\title{
Increased Resurgent Sodium Currents in Nav1.8 Contribute to Nociceptive Sensory Neuron Hyperexcitability Associated with Peripheral Neuropathies
}

\author{
Yucheng Xiao, ${ }^{1}$ CCindy Barbosa, ${ }^{2}$ Zifan Pei, ${ }^{1,2}{ }^{\oplus}$ Wenrui Xie, ${ }^{3}{ }^{\oplus}$ Judith A. Strong, ${ }^{3}$ Jun-Ming Zhang, ${ }^{3}$ \\ and $\odot$ Theodore R. Cummins ${ }^{1,2}$ \\ ${ }^{1}$ Department of Biology, School of Science, Indiana University-Purdue University Indianapolis, Indianapolis, Indiana 46202, ${ }^{2}$ Department of Pharmacology \\ and Toxicology, Indiana University School of Medicine, Indianapolis, Indiana 46202, and ${ }^{3}$ Department of Anesthesiology, University of Cincinnati, \\ Cincinnati, Ohio 45267
}

\begin{abstract}
Neuropathic pain is a significant public health challenge, yet the underlying mechanisms remain poorly understood. Painful small fiber neuropathy (SFN) may be caused by gain-of-function mutations in Nav1.8, a sodium channel subtype predominantly expressed in peripheral nociceptive neurons. However, it is not clear how Nav1.8 disease mutations induce sensory neuron hyperexcitability. Here we studied two mutations in Nav1.8 associated with hypersensitive sensory neurons: G1662S reported in painful SFN; and T790A, which underlies increased pain behaviors in the Possum transgenic mouse strain. We show that, in male DRG neurons, these mutations, which impair inactivation, significantly increase TTX-resistant resurgent sodium currents mediated by Nav1.8. The G1662S mutation doubled resurgent currents, and the T790A mutation increased them fourfold. These unusual currents are typically evoked during the repolarization phase of action potentials. We show that the T790A mutation greatly enhances DRG neuron excitability by reducing current threshold and increasing firing frequency. Interestingly, the mutation endows DRG neurons with multiple early afterdepolarizations and leads to substantial prolongation of action potential duration. In DRG neurons, siRNA knockdown of sodium channel $\beta 4$ subunits fails to significantly alter T790A current density but reduces TTX-resistant resurgent currents by $56 \%$. Furthermore, DRG neurons expressing T790A channels exhibited reduced excitability with fewer early afterdepolarizations and narrower action potentials after $\beta 4$ knockdown. Together, our data demonstrate that open-channel block of TTX-resistant currents, enhanced by gain-of-function mutations in Nav1.8, can make major contributions to the hyperexcitability of nociceptive neurons, likely leading to altered sensory phenotypes including neuropathic pain in SFN.
\end{abstract}

Key words: beta subunit; hyperexcitability; pain; resurgent; sodium channel

Significance Statement

This work demonstrates that two disease mutations in the voltage-gated sodium channel $\mathrm{Na}_{\mathrm{v}} 1.8$ that induce nociceptor hyperexcitability increase resurgent currents. Nav1.8 is crucial for pain sensations. Because resurgent currents are evoked during action potential repolarization, they can be crucial regulators of action potential activity. Our data indicate that increased Nav1.8 resurgent currents in DRG neurons greatly prolong action potential duration and enhance repetitive firing. We propose that Nav1.8 open-channel block is a major factor in Nav1.8-associated pain mechanisms and that targeting the molecular mechanism underlying these unique resurgent currents represents a novel therapeutic target for the treatment of aberrant pain sensations.

\section{Introduction}

Hyperactivity of small-diameter sensory neurons is frequently associated with neuropathic pain. Indeed, painful small fiber neuropathy (SFN) may be caused by abnormal hyperexcitability

Received Feb. 19, 2018; revised 0ct. 22, 2018; accepted Nov. 25, 2018.

Author contributions: Y.X. wrote the first draft of the paper; W.X., J.A.S., and J.-M.Z. edited the paper; Y.X., W.X., J.A.S., J.-M.Z., and T.R.C. designed research; Y.X., C.B., and Z.P. performed research; Y.X., C.B., Z.P., and T.R.C. analyzed data; T.R.C. wrote the paper. of nociceptive sensory neurons. However, the underlying mechanisms remain poorly understood, contributing to an unmet medical need. Recent studies on families with SFN indicate that, in $\sim 35 \%$ of patients, hereditary mutations in two genes SCN9A 
(Nav1.7) and SCN1OA (Nav1.8) are involved (Faber et al., 2012a; Hoeijmakers et al., 2012; Themistocleous et al., 2014).

Nav1.7 and Nav1.8 are preferentially expressed in nociceptive sensory neurons and are crucial contributors to neuropathic pain (Akopian et al., 1999; Cummins et al., 2007; Dib-Hajj et al., 2007). Although they share $>75 \%$ sequence similarity, they exhibit several unique biophysical properties. For example, Nav1.7 undergoes slower recovery from inactivation and slower closedstate inactivation (Cummins et al., 1998). Nav1.8 exhibits depolarized voltage dependencies and slower open-channel kinetics (Akopian et al., 1996). These unique biophysical properties help determine the distinct roles they play in action potential generation. Whereas Navl.7 is essential for setting voltage threshold, Nav1.8 is a major contributor to the rising phase (Cummins et al., 2007). Mutations identified in patients with painful SFN typically endow sodium channels with a hyperpolarized voltage dependence of activation and/or impaired inactivation kinetics, therefore facilitating the generation and repetitive firing of action potentials (Cummins et al., 2004; Fertleman et al., 2006; Faber et al., 2012b; Huang et al., 2013).

Resurgent currents mediated by sodium channels represent another important factor that influences neuronal excitability. In contrast to classic sodium currents elicited by depolarization, resurgent currents are unusual currents typically evoked during the repolarization phase of action potentials (Raman and Bean, 1997) by an open-channel blocker (Bant and Raman, 2010). Resurgent currents have been observed in multiple neuronal populations (Afshari et al., 2004; Cummins et al., 2005; Enomoto et al., 2006; Kim et al., 2010), and can promote generation of highfrequency action potential firing (Raman and Bean, 1997; Xie et al., 2016). Nav1.6 is the major carrier of resurgent current in DRG neurons, but other TTX-sensitive (TTX-S) sodium channel subtypes (e.g., Nav1.7) also exhibit an intrinsic ability to generate resurgent currents (Grieco and Raman, 2004; Cummins et al., 2005; Jarecki et al., 2010; Patel et al., 2015). Intriguingly, this ability is augmented under conditions that slow the rate of fast inactivation (Grieco and Raman, 2004; Jarecki et al., 2010). Our recent studies demonstrate that small-sized DRG neurons produce TTX-resistant (TTX-R) resurgent currents, which might be mediated by Nav1.8-like channels (Tan et al., 2014). Compared with Nav1.7-mediated resurgent currents, the TTX-R resurgent currents display much slower kinetics and are produced at more positive potentials. Computer simulations indicate that increased Nav1.7-mediated resurgent currents are important for inducing high-frequency action potential firing in nociceptive neurons in paroxysmal extreme pain disorder. By contrast, the influences of abnormal Nav1.8-mediated resurgent currents largely remain unknown. Given the distinct roles that specific sodium channel isoforms play in DRG action potential generation, it is of special interest to uncover whether abnormal Nav1.8-mediated resurgent currents might influence nociceptive neuron excitability in painful SFN and other painful conditions.

T790A and G1662S are Nav1.8 mutations identified in the Possum transgenic mouse strain and humans with painful SFN, respectively (Blasius et al., 2011; Han et al., 2014). Previous studies have shown that both of these mutations impair fast inactivation. We hypothesized that this impaired fast inactivation would lead to enhanced resurgent currents that increase action potential

Correspondence should be addressed to Theodore R. Cummins at trcummin@iupui.edu.

https://doi.org/10.1523/JNEUROSCI.0468-18.2018

Copyright $\odot 2019$ the authors $\quad 0270-6474 / 19 / 391540-12 \$ 15.00 / 0$ firing in sensory neurons. In this study, we found that these mutations did indeed significantly increase Nav1.8-mediated resurgent currents when expressed in rat small-diameter DRG neurons. We show that increased Nav1.8-mediated resurgent currents substantially broaden action potentials, induce multiple early afterdepolarizations (EADs), and increase firing frequency, therefore greatly enhancing excitability of nociceptive neurons. We propose that increased resurgent currents induced by gainof-function mutations in Nav1.8 contribute to enhanced hyperexcitability of nociceptive neurons that underlies altered sensory phenotypes, including neuropathic pain in SFN.

\section{Materials and Methods}

Sodium channel constructs and mutagenesis. The cDNA constructs encoding the mouse Nav1.8 (mNav1.8) and human Nav1.8 (hNav1.8) were synthesized as codon-optimized cDNA by GenScript and subcloned into a pcDNA3.1 $(+)$ expression vector. Two mNav1.8 mutations, T790A and G1663S, the second equal to G1662S in hNav1.8, as well as the G1662S mutation in hNav1.8 were constructed using the QuikChange XL (Stratagene) mutagenesis kit following the manufacturer's instructions (Stratagene). Mutations were confirmed by sequencing.

Cell culture and transfection. Rat DRG neurons were acutely dissociated and cultured according to the procedure described previously (Cummins et al., 2000). Briefly, young adult male Sprague Dawley rats, in adherence with animal procedures approved by the Indiana University School of Medicine and the School of Science Institutional Animal Care and Use Committees, were killed by carbon dioxide overexposure followed by decapitation. All DRGs or just lumbar DRGs (L4-L5) were removed quickly from the spinal cord and then incubated in DMEM containing collagenase $(1 \mathrm{mg} / \mathrm{ml})$ and protease $(1 \mathrm{mg} / \mathrm{ml})$. After the ganglia were triturated in DMEM supplemented with 10\% FBS, cells were plated on glass coverslips coated with poly-D-lysine and laminin. Cultures were maintained at $37^{\circ} \mathrm{C}$ in a $5 \% \mathrm{CO}_{2}$ incubator. The Helios Gene Gun (Bio-Rad) was used to transiently cotransfect rat DRG neurons, as described previously (Herzog et al., 2003; Dib-Hajj et al., 2009; Jarecki et al., 2010). Cells were cotransfected with a plasmid encoding the recombinant VGSC and an internal ribosome entry site-EGFP (IRESEGFP) vector plasmid that also encoded a Nav1.8 shRNA targeting the rat Nav1.8 (Mikami and Yang, 2005) but not the codon optimized mNav1.8 or hNav1.8 sequences. Under control conditions, the endogenous Nav1.8-type currents have an average current density of $947 \pm 72 \mathrm{pA} / \mathrm{pF}(n=70)$ and the Nav1.8 shRNA reduces endogenous Nav1.8-type current amplitudes in DRG neurons by $98 \%$ (Jarecki et al., 2010).

Electrophysiological recordings. DRG recordings were obtained from cells $2 \mathrm{~d}$ after transfection. The Nav1.8 current density was not significantly different from endogenous Nav1.8-type current density. Transfected cells were selected for recordings based on their expression of EGFP. Whole-cell patch-clamp recordings were performed at room temperature $\left(\sim 21^{\circ} \mathrm{C}\right)$ and $34^{\circ} \mathrm{C}$ using an EPC-10 amplifier and the Pulse program (HEKA Electronics). The temperature was increased to $34^{\circ} \mathrm{C}$ using a bipolar temperature controller (CL-100, Harvard Apparatus), a quick exchange heated/cooled platform for $35 \mathrm{~mm}$ Petri dishes (QE1HC, Warner Instruments) equipped with a thermal cooling module (TCM-1, Warner Instruments). Final temperatures were confirmed using a digital thermometer (Thermo Fisher Scientific).

For voltage-clamp recordings, fire-polished electrodes $(1.0-2.0 \mathrm{M} \Omega$ ) were fabricated from $1.7 \mathrm{~mm}$ capillary glass using a P-97 or P-1000 puller (Sutter Instruments), and the tips were coated with sticky wax (KerrLab) to minimize capacitive artifacts and enhance series resistance compensation. The pipette solution contained the following (in mM): $140 \mathrm{CsF}, 1.1$ EGTA, $10 \mathrm{NaCl}$, and 10 HEPES, pH 7.3. The bathing solution was as follows (in $\mathrm{mm}$ ): $130 \mathrm{NaCl}, 30$ TEA chloride, $1 \mathrm{MgCl}_{2}, 3 \mathrm{KCl}, 1 \mathrm{CaCl}_{2}$, $0.05 \mathrm{CdCl}_{2}, 10 \mathrm{HEPES}$, and 10 D-glucose, $\mathrm{pH} 7.3$ (adjusted with $\mathrm{NaOH}$ ). TTX (500 nM) was added to the bath solution to block endogenous TTX-S currents in DRG neurons. The liquid junction potential for these solutions was $<8 \mathrm{mV}$; data were not corrected to account for this offset. The pipette potential was zeroed before contacting the cell. After estab- 
lishing the whole-cell recording configuration, the resting potential was held at $-80 \mathrm{mV}$ for $5 \mathrm{~min}$ to allow adequate equilibration between the micropipette solution and the cell interior. Linear leak subtraction, based on resistance estimates from $4-5$ hyperpolarizing pulses applied before a depolarizing test potential, was used for all voltage-clamp recordings. Membrane currents were usually filtered at $5 \mathrm{kHz}$ and sampled at $20 \mathrm{kHz}$. Voltage errors were minimized using $80 \%-93 \%$ series resistance compensation, and the capacitance artifact was canceled using the computer-controlled circuitry of the patch-clamp amplifier. To avoid contamination from window currents, resurgent current was obtained by subtracting persistent current from the current measured after $30 \mathrm{~ms}$ into the repolarizing pulse. Peak resurgent current at each test potential was normalized to the transient current with maximal amplitude (obtained from the $I-V$ protocol). Normalized resurgent current amplitude was plotted as a function of voltage.

For current-clamp recordings, fire-polished electrodes $(4.0-5.0 \mathrm{M} \Omega$ ) were fabricated from $1.2 \mathrm{~mm}$ capillary glass using a P-97 or P-1000 puller (Sutter Instruments). The pipette solution contained the following (in mM): $140 \mathrm{KCl}, 5 \mathrm{MgCl}_{2}$, 5 EGTA, $2.5 \mathrm{CaCl}_{2}$, $4 \mathrm{ATP}, 0.3 \mathrm{GTP}$, and 10 HEPES, pH 7.3 (adjusted with $\mathrm{KOH}$ ). The bathing solution contained the following (in mM): $140 \mathrm{NaCl}, 1 \mathrm{MgCl}_{2}, 5 \mathrm{KCl}, 2 \mathrm{CaCl}_{2}, 10 \mathrm{HEPES}$, and 10 glucose, $\mathrm{pH} 7.3$ (adjusted with $\mathrm{NaOH}$ ). Neurons were allowed to stabilize for $3 \mathrm{~min}$ in the current-clamp mode before initiating current injections to measure action potential activity.

Procedure for in vivo injection of siRNA into the DRG. Male Sprague Dawley rats under isoflurane anesthesia were used for these procedures. siRNAs were prepared using cationic linear polyethylenimine-based transfection reagents (in vivo jetPEI, Polyplus Transfection, distributed by VWR Scientific). The siRNA reagents were "smartpools" consisting of four different siRNA constructs combined into 1 and were purchased from Dharmacon. The four siRNA sequences directed against rat Nav $\beta 4$ (catalog \#M-101002-01) were as follows: Construct 1, GGAUCGUGAAGAAUGAUAA; Construct 2, UCCAAGUGGUUGAUAAAUU; Construct 3, GCAAUACUCAGGCGAGAUG; and Construct 4, AAACAACUCUGCUACGAUC. The nontargeting control was directed against firefly luciferase gene (catalog \#D-001210-02). This luciferase sequence (UAAGGCUAUGAAGAGAUAC) is unrelated to the shRNA sequence recently reported to have extensive off-target effects in hippocampal neurons (Hasegawa et al., 2017). Aliquots of $3 \mu \mathrm{l}$ of containing siRNA/jetPEI mixture ( 80 pmol of siRNA) were injected into each L4 and L5 DRG on one side, through a small glass needle inserted close to the DRG as previously described (Xie et al., 2013; Barbosa et al., 2015). Three days after injection, ipsilateral L4 and L5 DRGs were harvested. Dissociated DRG neurons were examined by immunocytochemistry to verify knockdown, and another fraction was used for transfection and whole-cell patch clamp. A previous study using the same methodology showed that two separate individual Nav $\beta 4$ siRNA constructs from the smartpool reduced immunostaining and had similar behavioral effects as the smartpool (Xie et al., 2016), providing evidence against sequence-dependent off-target effects of the siRNA construct used (Jackson and Linsley, 2010). Indiana University School of Medicine Institutional Animal Care and Use Committee approved the animal protocols described.

Immunocytochemistry. To verify knockdown of Nav $\beta 4$ protein, L4 and L5 ipsilateral DRGs harvested and cultured from rats injected with nontargeting control and $\beta 4$-siRNA were examined $5 \mathrm{~d}$ after dissociation in parallel with whole-cell patch-clamp experiments. DRG neurons were fixed with $4 \%$ PFA (0.1 M phosphate buffer, $\mathrm{pH} 7.4$ ) for $20 \mathrm{~min}$ and washed in PBS. Cells were then permeabilized in 1\% Triton X-100 in PBS for $20 \mathrm{~min}$ at room temperature $\left(\sim 21^{\circ} \mathrm{C}\right)$, washed in PBS, blocked for $2 \mathrm{~h}$ ( $10 \%$ normal goat serum, $0.1 \%$ Triton X-100 in PBS) at room temperature, and washed with PBS. Cells were then incubated with polyclonal anti-Nav $\beta 4$ antibody (1:500, \#Ab80539, Abcam) diluted in blocking solution overnight at $4^{\circ} \mathrm{C}$. After additional PBS washes, cells were incubated with secondary antibody AlexaFluor-488 Goat Anti-Rabbit IgG (Molecular Probes, Invitrogen) in blocking solution at 1:1000 concentration for $2 \mathrm{~h}$ at room temperature. Coverslips were mounted in Prolong Gold Antifade (Invitrogen) and DRG neurons imaged using Axio Observer Z1 Widefield Microscope with a $20 \times$ objective (Carl Zeiss). Images were analyzed with NIS Elements Advance Research (Nikon) software, and corrected total cell fluorescence was calculated in Excel (Microsoft) by applying measurements obtained from image analysis using the following equations adapted from Barbosa et al. (2015):

$$
\begin{aligned}
C T C F=\begin{array}{c}
\text { Integrated } \\
\text { Density }
\end{array} & -(\text { Area of selected cell } \\
& \times \text { mean fluorescence of background readings })
\end{aligned}
$$

where

$$
\text { Integrated Density }=(\text { mean intensity } \times \text { area of the cell })
$$

Experimental design and statistical analysis. The acquisition of control and experimental data was randomized. Data were analyzed using the software programs PulseFit (HEKA) and Prism 5.0 (GraphPad Software). All data are shown as mean \pm SE. The number of separate experimental cells is presented as $n$. Channel conductance was calculated using the equation $G(\mathrm{Nav})=I /\left(V-V_{\text {rev }}\right)$ in which $I, V$, and $V_{\text {rev }}$ represent inward current value, membrane potential, and reversal potential, respectively. Statistical analysis was performed by Student's $t$ test and $\chi^{2}$ analysis, and $p<0.05$ indicated a significant difference.

\section{Results \\ The Possum mutation in Nav1.8 that slows fast inactivation increases TTX-R resurgent currents}

We first asked whether alteration of gating properties by the T790A mutation, originally characterized in the Possum mouse line, could be replicated in rat DRG neurons. Endogenous rat Nav1.8 currents are knocked down by $>98 \%$ under our experimental conditions (Jarecki et al., 2010) by the rat Nav1.8 targeting shRNA (Mikami and Yang, 2005). As shown in Figure 1A, when transiently expressed in rat DRG neurons, the Possum mutation substantially slowed down inactivation of mNav1.8 channels at room temperature $\left(21^{\circ} \mathrm{C}\right)$. Nav1.8 inactivation was well fit by two exponentials (Fig. $1 B$ ). At $10 \mathrm{mV}$, the fast-component time constant was $2.42 \pm 0.17 \mathrm{~ms}$ and $3.47 \pm 0.22 \mathrm{~ms}(p<0.001)$ for WT and T790A mNav1.8 channels, respectively, whereas the slow-component value was $11.19 \pm 1.07 \mathrm{~ms}$ and $17.78 \pm 1.62 \mathrm{~ms}$ $(p<0.005)$, respectively. The Possum mutation did not affect the voltage dependence of steady-state inactivation (Fig. 1C). These major observations are fully consistent with those characterized for Possum mouse DRG neurons. However, we also observed that the Possum mutation shifted the voltage dependence of mNav1.8 activation by $-11.9 \mathrm{mV}$ in rat DRG neurons at room temperature $\left(21^{\circ} \mathrm{C}\right)(\mathrm{WT},-12.3 \pm 0.3 \mathrm{mV}$; T790A, $-24.2 \pm 0.4 \mathrm{mV}$; Fig. $1 C)$. There was not a statistical difference in current density associated with mNav1.8 and T790A mutant channels; the average peak current density was $-1054 \pm 234 \mathrm{pA} / \mathrm{pF}$ and $-1230 \pm 249$ $\mathrm{pA} / \mathrm{pF}(p>0.5)$, respectively. It is important to note that these peak current densities are remarkably similar to that observed for rat Nav1.8 currents under control conditions $(-1063 \pm 128 \mathrm{pA} /$ $\mathrm{pF}, n=20)$.

We then examined the change in gating properties of mNav1.8 by the T790A mutation at $34^{\circ} \mathrm{C}$, a temperature close to physiological body temperature. As reported previously (Zimmermann et al., 2007), the increase in temperature did not evidently shift the voltage dependence of either steady-state mNav1.8 activation or inactivation (Fig. $1 C$ ) but accelerated the fast component of mNav1.8 inactivation kinetics (Fig. $1 A, B$; Table 1 ). At $34^{\circ} \mathrm{C}$, the possum mutation also significantly slowed down mNav1.8 inactivation (compared with WT mNav1.8) by increasing the fast-component time constant from $1.47 \pm 0.14$ $\mathrm{ms}$ to $2.26 \pm 0.23 \mathrm{~ms}(p<0.01)$ and the slow-component value from $9.38 \pm 0.68 \mathrm{~ms}$ to $13.45 \pm 0.94 \mathrm{~ms}(p<0.005)$, respectively. Moreover, as observed at $21^{\circ} \mathrm{C}$, the possum mutation did not 
significantly affect steady-state mNav1.8 inactivation at $34^{\circ} \mathrm{C}$ compared with WT but shifted the voltage dependence of mNav1.8 activation by $-14.6 \mathrm{mV}$ (WT, $-8.6 \pm 0.5 \mathrm{mV}$; T790A, $-23.2 \pm 0.5 \mathrm{mV}$; Fig. $1 C$ ). It is important to note that, although some ion channels exhibit pronounced temperature sensitivity, our data support the conclusion of Zimmerman et al. (2007) that Nav1.8 inactivation properties are relatively resistant to temperature-induced changes.

Recently, we identified a slow TTX-R resurgent current in small-diameter DRG neurons that is predicted to be generated by Nav1.8 and that is predicted to modulate nociceptor excitability (Tan et al., 2014). The resurgent current mechanism may compete with channel classic fast inactivation (Cannon and Bean, 2010) and human disease mutations that impair fast inactivation of TTX-S channels substantially increase resurgent currents (Jarecki et al., 2010). We thus tested whether mNav1.8 has intrinsic ability to generate TTX-R resurgent currents and whether the Possum mutation would alter Nav1.8mediated resurgent current generation. It is important to note that, when Nav1.8 channels are expressed in the ND7/23 heterologous cell line and interrogated with a resurgent current protocol (Fig. 2A), classic tail currents are elicited but not resurgent currents. However, mNav1.8 channels transfected into DRG neurons generate robust resurgent currents that are nearly identical to endogenous TTX-R slow resurgent currents previously described (Fig. 2C). We used a $20 \mathrm{~ms}$ initial depolarization in the resurgent current protocol. Although Nav1.8 inactivation is often incomplete with $20 \mathrm{~ms}$ depolarizations, we used this duration because it allows direct comparisons to previous studies on TTX-S resurgent currents, grossly mimics nociceptor action potential durations, and minimizes the confounding influences of slow inactivation (Blair and Bean, 2003). As illustrated by the superimposed currents in Figure 2B, the classic Nav1.8 tail currents observed in ND7/23 cells arise nearly instantaneously and decay rapidly, whereas the slow resurgent currents observed with DRG mNav1.8 transfection have much slower onset and decay kinetics. These slow resurgent currents are observed with mNav1.8 transfection but were not observed when Nav1.4, Nav1.5, Nav1.6, or Nav1.7 channels are transfected into Nav1.8-shRNA treated DRG neurons (Jarecki et al., 2010). We find that, in the majority of mNav1.8transfected rat DRG neurons ( 7 of 8 cells at $21^{\circ} \mathrm{C}$; 16 of 20 cells at $34^{\circ} \mathrm{C}$ ), TTX-R resurgent currents were inducible when the membrane was repolarized to voltages ranging from 5 to $-40 \mathrm{mV}$. The currents peaked at -10 to $-15 \mathrm{mV}$ (Fig. $1 C$ ). The time to peak and the decay time constant for the WT currents elicited at $-15 \mathrm{mV}$ were $45.1 \pm 8.6 \mathrm{~ms}$ and $618.3 \pm 81.2 \mathrm{~ms}$ at $21^{\circ} \mathrm{C}(12.0 \pm 2.3 \mathrm{~ms}$ and $532.8 \pm 51.9 \mathrm{~ms}$ at $34^{\circ} \mathrm{C}$ ), respectively. These data indicate that, while resurgent current decay shows little temperature dependence, the onset of resurgent currents is substantially faster at warmer temperatures $(p<0.005)$. These mNav1.8 resurgent currents exhibited a more depolarized voltage dependence of activation compared with the fast resurgent currents mediated by other Nav subtypes (including Nav1.6 and Nav1.7), which peak at $\sim-40 \mathrm{mV}$ (Jarecki et al., $21^{\circ} \mathrm{C}$

$+10 \mathrm{mV}, 50 \mathrm{~ms}$

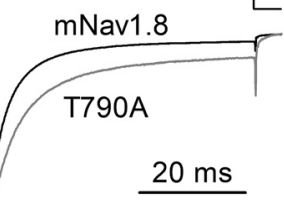
$\mathrm{mNav1.8}$ ○ $\mathrm{T}$ T790A ${ }_{0}^{* *}$

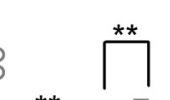
(1)

Figure 1. The Possum T790A mutation alters gating properties of mNav1.8 in rat DRG neurons at $21^{\circ} \mathrm{C}$ and $34^{\circ} \mathrm{C}$. $A$, The Possum mutation significantly slowed fast inactivation. Left, Right, Typical current traces were elicited by a $50 \mathrm{~ms}$ depolarizing potential of . dependent activation, but not steady-state inactivation, to more negative potentials. Steady-state inactivation was estimated as a protocol in which currents were induced by a $20 \mathrm{~ms}$ depolarizing potential of $0 \mathrm{mV}$ following a 500 $\mathrm{mV}(n=13) ; \mathrm{T} 790 \mathrm{~A},-24.2 \pm 0.4 \mathrm{mV}(n=16) . V_{1 / 2}$ for inactivation: $\mathrm{mNav1} 1.8,-32.7 \pm 0.5 \mathrm{mV}(n=9) ; \mathrm{T} 790 \mathrm{~A},-36.5 \pm 0.7$ $\mathrm{mV}(n=13)$. At $34^{\circ} \mathrm{C}$ (square symbols), $V_{1 / 2}$ for activation: $\mathrm{mNav1} .8,-8.6 \pm 0.5 \mathrm{mV}(n=16) ; \mathrm{T} 790 \mathrm{~A},-23.2 \pm 0.5 \mathrm{mV}(n=$ , $-27.7 \pm 1.6 \mathrm{mV}(n=14) ; \mathrm{T790A},-33.1 \pm 0.9 \mathrm{mV}(n=14) .{ }^{* *} p<0.01,{ }^{* * *} p<0.001$

Table 1. Inactivation time constants of mNav1.8 and $\mathrm{T} 90 \mathrm{~A}^{a}$

\begin{tabular}{|c|c|c|c|c|c|c|}
\hline \multirow{2}{*}{$\begin{array}{l}\text { Temperature } \\
\left({ }^{\circ} \mathrm{C}\right)\end{array}$} & \multicolumn{3}{|l|}{ mNav1.8 } & \multicolumn{3}{|l|}{ T790A } \\
\hline & $\tau_{\text {fast }}$ (ms) & $\tau_{\text {slow }}(\mathrm{ms})$ & $n$ & $\tau_{\text {fast }}(\mathrm{ms})$ & $\tau_{\text {slow }}(\mathrm{ms})$ & $n$ \\
\hline 21 & $2.42 \pm 0.17$ & $11.19 \pm 1.07$ & 15 & $3.47 \pm 0.22$ & $17.78 \pm 1.62$ & 19 \\
\hline 34 & $1.47 \pm 0.14$ & $9.38 \pm 0.68$ & 21 & $2.26 \pm 0.23$ & $13.45 \pm 0.94$ & 19 \\
\hline$p$ & $<0.001$ & $>0.05$ & & $<0.001$ & $<0.001$ & \\
\hline
\end{tabular}

${ }^{a}$ Sodium currents were elicited by a $50 \mathrm{~ms}$ depolarizing potential of $10 \mathrm{mV}$ from a holding potential of $-80 \mathrm{mV}$ (see Fig. 1A). Data are mean \pm SEM.

2010). WT mNav1.8-mediated resurgent current amplitude (measured as indicated in Fig. 2B, double-headed arrow) was $2.1 \pm 0.4 \%$ of the peak transient current at $21^{\circ} \mathrm{C}$ and $2.2 \pm 0.3 \%$ at $34^{\circ} \mathrm{C}$ (Fig. $2 F, H)$. TTX-R resurgent currents could be elicited in almost all T790A-transfected rat DRG neurons ( 9 of 9 cells at $21^{\circ} \mathrm{C} ; 13$ of 15 cells at $34^{\circ} \mathrm{C}$ ). The largest resurgent current was attained at $-15 \mathrm{mV}$ to $-20 \mathrm{mV}$ (Fig. 2C,D). Interestingly, although the Possum mutation did not significantly change the voltage at which TTX-R resurgent currents peaked, the plot of normalized resurgent currents shows that there is substantially increased current at voltages between -30 and $-45 \mathrm{mV}$ (Fig. 2E, G). The Possum mutation was found to induce a greater than fourfold increase in relative resurgent current amplitude. The peak resurgent current amplitude was $10.2 \pm 0.8 \%$ of the peak transient current at $21^{\circ} \mathrm{C}$ and $9.0 \pm 0.9 \%$ at $34^{\circ} \mathrm{C}$ (Fig. $2 F, H)$. As is illustrated in Figure $2 B$, resurgent current amplitude is measured from the peak of the slow onset phase to the residual current at the end of the $1 \mathrm{~s}$ repolarization step. Accurate measurement of resurgent current amplitude is complicated by the slow kinetics of Nav1.8 currents and by the relatively depolarized voltage dependence of Nav1.8 resurgent currents. 
A

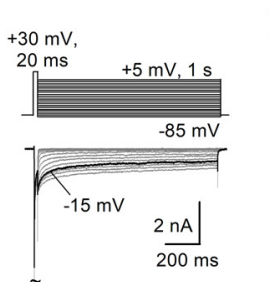

B
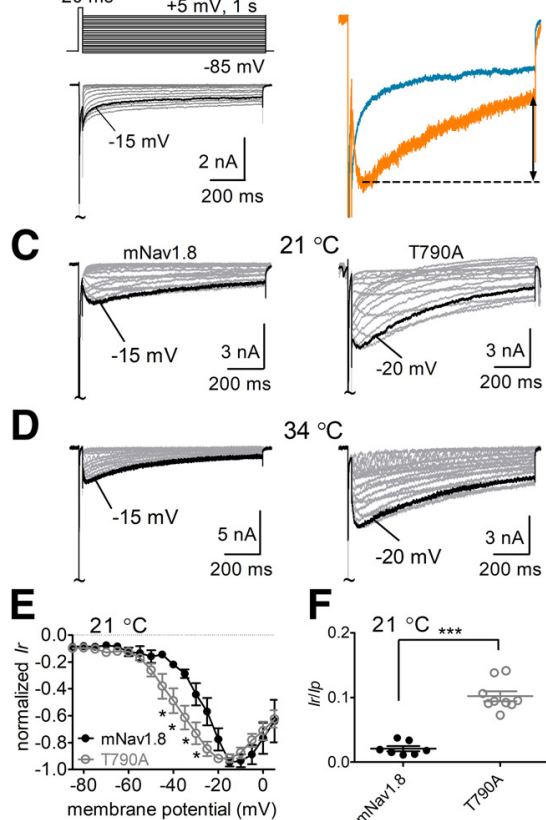

$34^{\circ} \mathrm{C}$

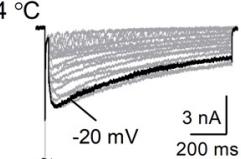

$\mathbf{F}$

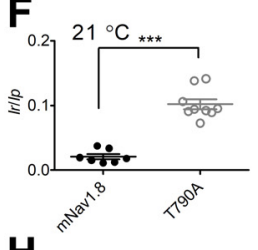

G
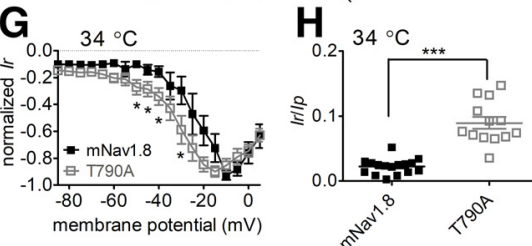

Figure 2. The Possum T790A mutation enhances mNav1.8 resurgent currents. $\boldsymbol{A}$, Representative tail currents induced by a resurgent current protocol (top left) recorded from ND7/23 cells expressing Nav1.8 channels. The resurgent current protocol is a two-step protocol, in which the cell membrane is initially depolarized to $30 \mathrm{mV}$ for $20 \mathrm{~ms}$, followed by $1 \mathrm{~s} \mathrm{hyperpolarizing} \mathrm{steps}$ to potentials ranging from 5 to $-85 \mathrm{mV}$. $\boldsymbol{B}$, Comparison of Nav1.8 tail current (blue trace) with a mNav1.8 resurgent current recorded a DRG neuron (orange trace) elicited with a $-15 \mathrm{mV}$ hyperpolarizing voltage step. Dashed line and double headed arrow indicate how resurgent current amplitude was estimated. $C, D$, Representative resurgent currents recorded from DRG neurons expressing mNav1.8 (left) and T790A (right) channels at $21^{\circ} \mathrm{C}(\boldsymbol{C})$ and $34^{\circ} \mathrm{C}(\boldsymbol{D}) . \boldsymbol{E}$, Comparison of normalized resurgent current-voltage relationships of mNav1.8-mediated $(n=7)$ and T790A-mediated $(n=9)$ resurgent currents at $21^{\circ} \mathrm{C}$. $\boldsymbol{F}$, Summary of ratio resurgent currents at $21^{\circ} \mathrm{C}$. Filled and open symbols represent mNav1.8 and T790A mutant channels, respectively. $\mathbf{G}$, Comparison of normalized resurgent current-voltage relationships of mNav1.8-mediated $(n=15)$ and T790A-mediated $(n=13)$ resurgent currents at $34^{\circ} \mathrm{C} . \boldsymbol{H}$, Summary of ratio resurgent currents at $34^{\circ} \mathrm{C}$. In $\boldsymbol{A}-\boldsymbol{H}$, Cells were held at $-80 \mathrm{mV}$, and all recordings were performed in the presence of $500 \mathrm{~nm}$ TTX.E, G, Filled and open symbols represent mNav1.8 and T790A mutant channels, respectively. Data points are mean \pm SE. ${ }^{*} p<$ $0.05,{ }^{* * *} p<0.001$.

\section{The Possum mutation in Nav1.8 substantially increases sensory neuron excitability}

Raman et al. (1997) reported that in cerebellar Purkinje neurons, resurgent currents are required for generating high-frequency action potentials. Therefore, we next asked how increasing Nav1.8-mediated resurgent currents might modulate excitability of nociceptive neurons at room temperature and physiological body temperature. In this study, 20 small-sized neurons with mNav1.8 channels and 24 with T790A mutant channels were patched and recorded at $21^{\circ} \mathrm{C}$ in the whole-cell current-clamp mode, and 22 with mNav1.8 channels and 22 with T790A mutant channels were studied at $34^{\circ} \mathrm{C}$. Our recordings indicated that the Possum mutation did not change resting membrane potential $(\mathrm{mNav1.8},-57.2 \pm 0.8 \mathrm{mV}$ vs T790A, $-58.3 \pm 1.6 \mathrm{mV}, p>$
$0.05)$ but increased the number of neurons that fired spontaneously. At $21^{\circ} \mathrm{C}$, spontaneous firing was observed in $\sim 13 \%$ of mNav1.8-transfected neurons ( 3 of 23 cells), which is close to the percentage $(14 \%)$ of neurons transfected with rat Nav1.8 channels that exhibit spontaneous activity (Han et al., 2014). In contrast, the expression of T790A mutant channels was associated with an almost threefold increase in the proportion of spontaneous firing neurons, with 57 percentage of DRG neurons ( 13 of 23 cells) exhibiting spontaneous activity (Fig. $3 A, B)$. Increasing temperature may induce a higher proportion of spontaneous firing DRG neurons. At $34^{\circ} \mathrm{C}$, the proportion was $27.3 \%$ ( 6 of 22 cells) for mNav1.8 and $63.6 \%$ ( 14 of 22 cells) for T790A, respectively. The difference in spontaneous activity was significant at both $21^{\circ} \mathrm{C}$ and $34^{\circ} \mathrm{C}\left(\chi^{2}\right.$ analysis; $p<$ $0.02)$.

The Possum mutation substantially broadened the duration of single action potentials elicited by a short (1 ms) current injection. Although small resurgent currents were inducible in the majority of WT mNav1.8-transfected DRG neurons, all of the action potentials elicited in these neurons were comparatively narrow at both temperatures examined (Fig. 3C,D). As described for nociceptive neurons by other studies (Djouhri et al., 1998; López de Armentia et al., 2000; Ritter et al., 2015), these WT action potentials exhibit a prominent shoulder during the repolarization phase. WT mNav1.8-transfected DRG neurons generated action potentials with an average duration (measured at the base) of $16.0 \pm 0.7 \mathrm{~ms}$ at room temperature and $11.0 \pm 1.0 \mathrm{~ms}$ at $34^{\circ} \mathrm{C}$ (Fig. 2D). The duration ranged from 10.1 to $23.1 \mathrm{~ms}$ at $21^{\circ} \mathrm{C}$ and from 4.8 to $24.7 \mathrm{~ms}$ at $34^{\circ} \mathrm{C}$. The durations measured at $34^{\circ} \mathrm{C}$ are generally consistent with those measured at the base previously in rat C-nociceptors in vivo (Fang et al., 2005). By contrast, T790A-transfected neurons produced strikingly prolonged action potentials with an average duration of $163.5 \pm 44.8 \mathrm{~ms}$ at $21^{\circ} \mathrm{C}$ and $575.4 \pm 134.0 \mathrm{~ms}$ at $34^{\circ} \mathrm{C}$. The duration ranged widely from 16.1 to $702.4 \mathrm{~ms}$ at $21^{\circ} \mathrm{C}$; and, surprisingly, the duration varied from 7.9 to $1737 \mathrm{~ms}$ at $34^{\circ} \mathrm{C}$. This suggests that the Possum mutation substantially broadened action potentials in nociceptive neurons by $>10$-fold. It is worth noting that multiple EADs were present during the plateau phase of action potentials in most T790A-transfected neurons (Fig. 3C). A similar phenomenon has also been observed with long-QT3 syndrome mutations, which impair fast inactivation of cardiac sodium channels and cause fatal arrhythmias (Nuyens et al., 2001; Song et al., 2012). EADs were not observed in any WT mNav1.8-transfected DRG neuron. Compared with WT mNav1.8-transfected DRG neurons, T790A-transfected DRG neurons exhibited a lower current threshold for action potential initiation (Fig. 3E). The Possum mutation greatly increased firing frequency elicited by a long $(2 \mathrm{~s})$ current injection. As shown in Figure $3 F, G, 100$ pA current injection failed to induce action potentials in the majority of WT mNav1.8-transfected DRG neurons ( 18 of 22 cells at $21^{\circ} \mathrm{C} ; 15$ of 21 cells at $34^{\circ} \mathrm{C}$ ). Even when injected with $200 \mathrm{pA}$ current, firing frequency in most WT-transfected DRG neurons ( 17 of 22 cells at $21^{\circ} \mathrm{C} ; 20$ of 21 cells at $34^{\circ} \mathrm{C}$ ) was not $>0.5 \mathrm{~Hz}$ (Fig. $3 \mathrm{H}$ ). In contrast, the mean firing frequency of T790A-transfected DRG neurons by the same current injections was $3.3 \pm 0.8 \mathrm{~Hz}$ and $4.7 \pm 1.1 \mathrm{~Hz}$ at $21^{\circ} \mathrm{C}\left(2.1 \pm 0.6 \mathrm{~Hz}\right.$ and $5.1 \pm 1.6 \mathrm{~Hz}$ at $\left.34^{\circ} \mathrm{C}\right)$, respectively. Although the increase in action potential number induced by $200 \mathrm{pA}$ current injection is not statistically significant at $34^{\circ} \mathrm{C}$, the action potentials had a much longer duration for T790A-transfected neurons than for mNav1.8-transfected neurons (Fig. 3F). Among the 21 T790A-transfected neurons, five only generated one action potential, and the action potential had 
A
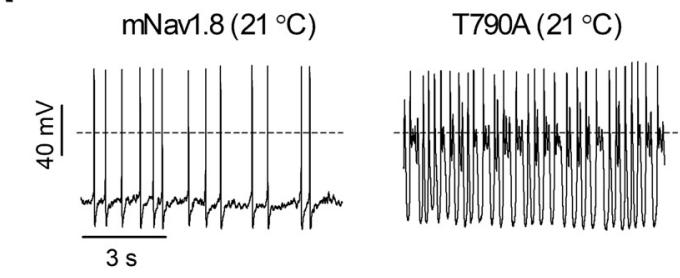

C

mNav1.8 T790A

$\left(21^{\circ} \mathrm{C}\right) \quad\left(21^{\circ} \mathrm{C}\right)$

(i)

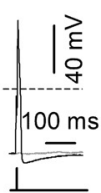

(ii)
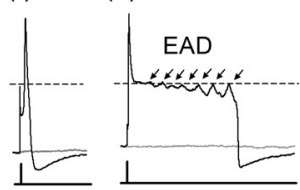

$\mathrm{mNav1} .8$
$\left(34^{\circ} \mathrm{C}\right)$

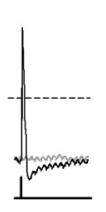

$\operatorname{mNav1} .8\left(34^{\circ} \mathrm{C}\right)$

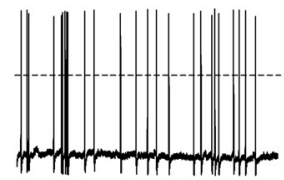

$\operatorname{T790A}\left(34^{\circ} \mathrm{C}\right)$

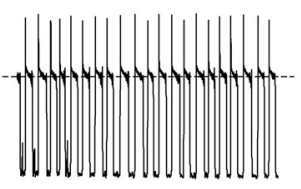

B

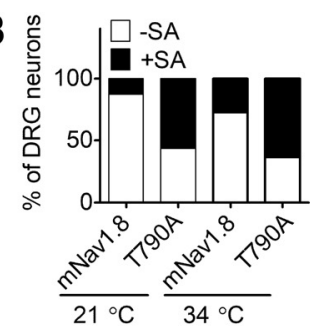

F
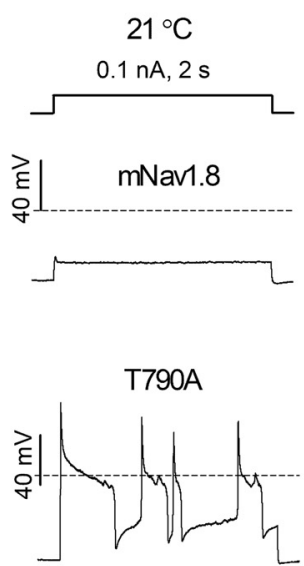

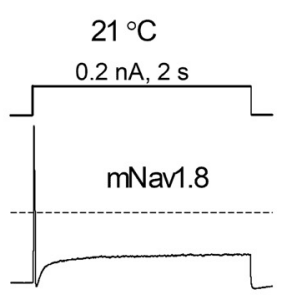

(i) $\left(34^{\circ} \mathrm{C}\right)$

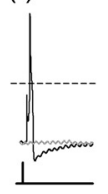

(ii)
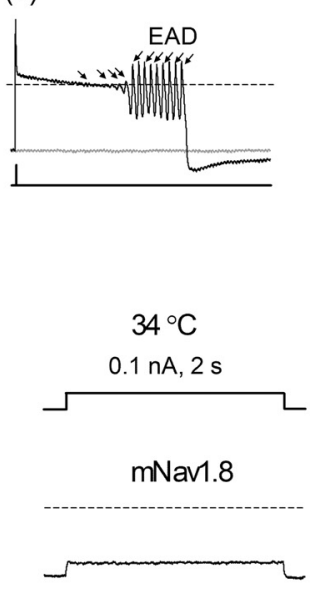
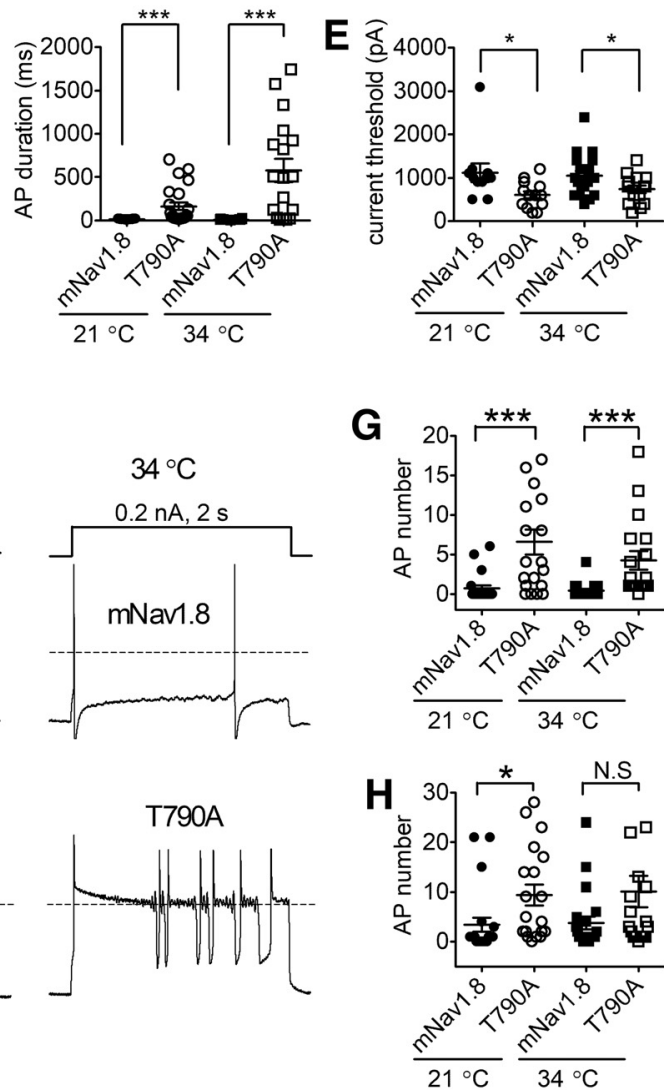

Figure 3. The Possum mutation upregulated excitability of small-sized DRG neurons at $21^{\circ} \mathrm{C}$ and $34^{\circ} \mathrm{C}$. $A$, Typical spontaneous firing recorded from mNav1.8- or T790A-transfected DRG neurons. $B$, Bar graph represents the percentage of DRG neurons with (black) or without (white) spontaneous firing. + SA, Spontaneous activity; $-S A$, no spontaneous activity. C, Representative single action potentials elicited by a $1 \mathrm{~ms}$ current injection in DRG neurons transfected with mNav1.8 or T790A mutant channels. Injected currents ranged from 0 to 3200 pA. $i$, ii, Two typical types of single action potentials on T790A-transfected neurons. Gray voltage traces represent resting membrane potential. EAD was indicated by arrows during the shoulder of the repolarization elicited in T790Atransfected neurons (right). $\boldsymbol{D}, \boldsymbol{E}$, Summary of action potential durations (APD90) and current thresholds. At $21^{\circ} \mathrm{C}$, the average $A P D 90$ s were $16.0 \pm 0.7 \mathrm{~ms}(\mathrm{mNav} 1.8, n=21)$ and $163.5 \pm 44.8 \mathrm{~ms}$ $(\mathrm{T} 790 \mathrm{~A}, n=23)$, respectively. The average current thresholds were $900.0 \pm 59.6 \mathrm{pA}$ (mNav1.8) and $454.5 \pm 60.9 \mathrm{pA}(\mathrm{T790A})$, respectively. At $34^{\circ} \mathrm{C}$, the average $A P D 90 \mathrm{~s}$ were $11.0 \pm 1.0 \mathrm{~ms}$ ( $\mathrm{mNav1.8,} n=22$ ) and $575.4 \pm 134.0 \mathrm{~ms}(\mathrm{~T} 790 \mathrm{~A}, n=18)$, respectively. The average current thresholds were $1047.0 \pm 111.2 \mathrm{pA}$ (mNav1.8) and 735.7 $\pm 90.6 \mathrm{pA}(\mathrm{T} 790 \mathrm{~A})$, respectively. $\boldsymbol{F}$, Representative trains of action potentials evoked by a 2 s injection of 100 or $200 \mathrm{pA}$ currents. $\boldsymbol{G}, \boldsymbol{H}$, Summary of the number of action potentials evoked during the $2 \mathrm{~s}$ injection. Filled and open circles represent mNav1.8 and T790A mutant channels, respectively. $A, C, F$, Dotted line indicates zero voltage level. ${ }^{*} p<0.05,{ }^{* * *} p<0.001$.

a $2 \mathrm{~s}$ long plateau phase at $\sim 0 \mathrm{mV}$ due to the firing of multiple EADs.

Knockdown of Nav $\beta 4$ reduced sensory neuron excitability by decreasing Nav1.8-mediated resurgent currents

Nav $\beta 4$ subunit is highly expressed in DRG neurons (Yu et al., 2003). There is evidence to support that its cytoplasmic tail functions as an open-channel blocker, which underlies the generation of resurgent currents (Grieco et al., 2005; Bant and Raman, 2010; Theile and Cummins, 2011; Barbosa et al., 2017). To confirm the roles of increased Nav1.8-mediated resurgent currents in nociceptive neuron hyperexcitability, we next asked whether resurgent current reduction by Nav $\beta 4$ knockdown would decrease the excitability of T790A-transfected DRG neurons. The following experiments were performed only at room temperature because the
T790A mutation similarly increased Nav1.8-mediated resurgent currents and upregulated DRG neuron excitability at $21^{\circ} \mathrm{C}$ and $34^{\circ} \mathrm{C}$ (Figs. 2, 3).

In adult rat L4 and L5 DRG neurons, $\mathrm{Nav} \beta 4$ siRNA treatment was found to depress expression of Nav $\beta 4$ proteins by $90 \%$ (Fig. $4 A, B)$. However, there was no statistical difference in T790A mutant channel current density (control, $-0.98 \pm 0.19 \mathrm{nA} / \mathrm{pF}$, $n=8$; Nav $\beta 4$ siRNA, $-0.78 \pm 0.17 \mathrm{nA} / \mathrm{pF}, n=13 ; p>0.05)$. $\mathrm{Nav} \beta 4$ knockdown did not significantly affect inactivation kinetics, the voltage dependence of activation, or steady-state inactivation of T790A mutant channels (Fig. 4C,D). Nav $\beta 4$ knockdown did not significantly alter the fraction of transfected DRG neurons that generated TTX-R resurgent currents (control, $100 \%, 8$ of 8 cells; Nav $\beta 4$ siRNA, $77 \%, 10$ of 13 cells) but substantially reduced the relative T790A resurgent current ampli- 

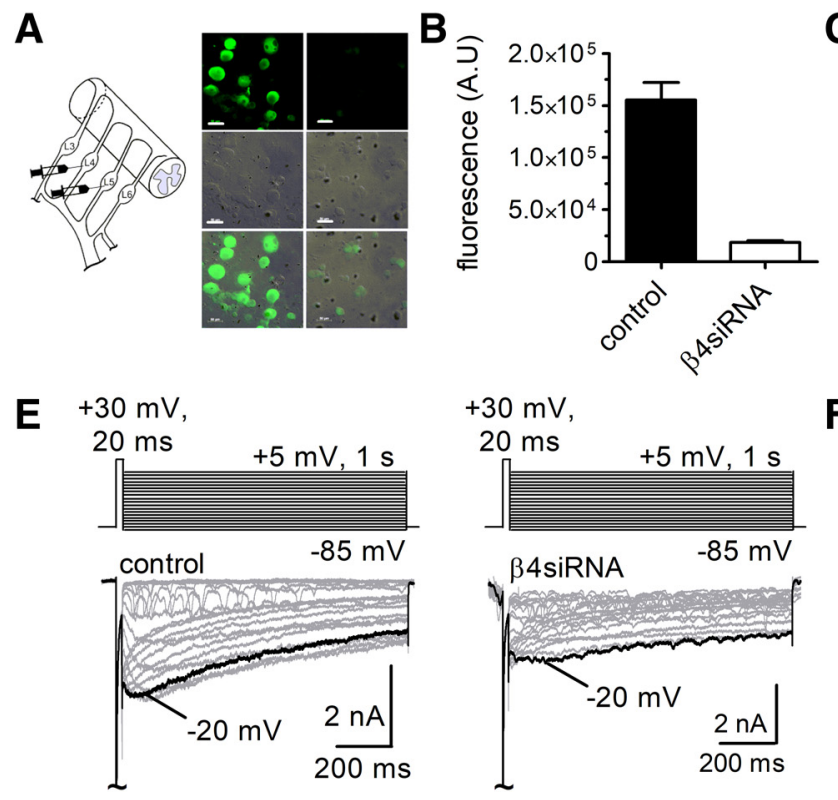

C

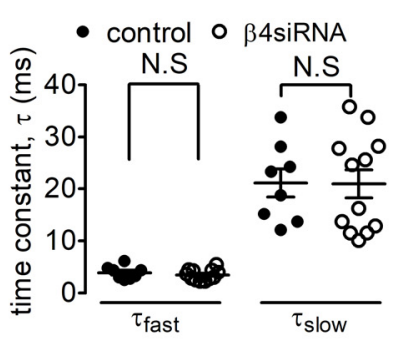

$F$

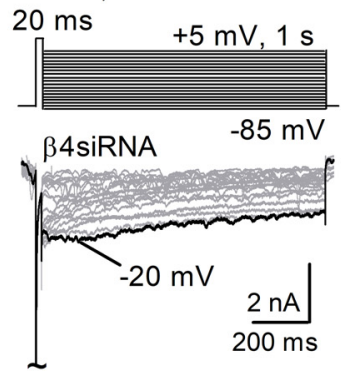

D

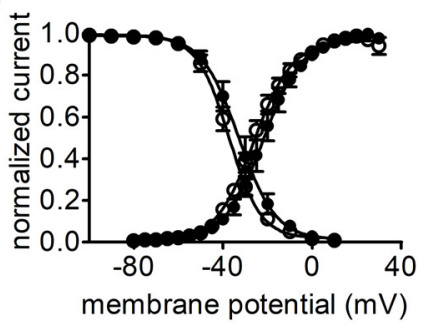

G

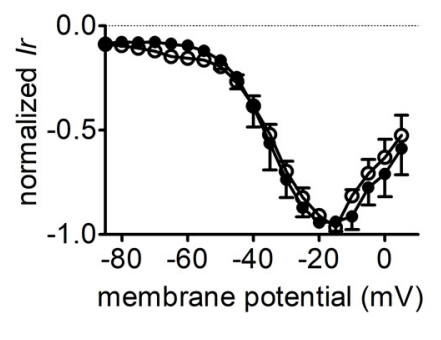

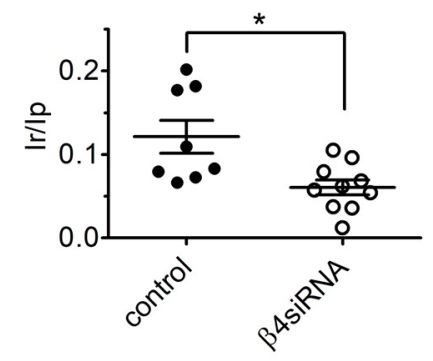

Figure 4. Knockdown of Nav $\beta 4$ reduced the ability of T790A mutant channels to generate resurgent currents in L4 - L5 DRG neurons. $\boldsymbol{A}$, Left, Diagram represents L4 - L5 DRGs injected by Nav $\beta 4$ siRNA. Right, Immunofluorescent reactions showed Nav $\beta 4$ subunit expression levels in DRG neurons, in which T790A mutant channels were not transfected. Scale bars, $50 \mu \mathrm{m}$. $\boldsymbol{B}$, Summary of fluorescence in L4-L5 DRG neurons with or without siRNA treatment. C, Knockdown of Nav $\beta 4$ did not significantly change either the fast component time constant or the slow component of inactivation kinetics. For $\tau_{\text {fast }}$ : control, $3.9 \pm 0.4 \mathrm{~ms}(n=8) ; \beta 4$ siRNA, $3.5 \pm 0.3 \mathrm{~ms}(n=12), p>0.1$. For $\tau_{\text {slow: }}$ : control, $21.1 \pm 2.7 \mathrm{~ms} ; \beta 4 \operatorname{siRNA}, 21.0 \pm 2.7 \mathrm{~ms}, p>0.1 . D$, Knockdown of Nav $\beta 4$ did not alter steady-state activation or inactivation of T790A mutant channels. $V_{1 / 2}$ for activation: control, $-21.8 \pm 0.7 \mathrm{mV}(n=7)$; $\beta 4$ siRNA, $-26.2 \pm 0.5 \mathrm{mV}(n=9)$. $V_{1 / 2}$ for inactivation: control, $-33.0 \pm 1.1 \mathrm{mV}(n=7) ; \beta 4$ siRNA, $-37.4 \pm 0.6 \mathrm{mV}(n=9)$. $\boldsymbol{E}$, Representative resurgent current traces for control and $\beta 4$ siRNA elicited by a two-step protocol as described in Figure $2 A$. Neurons were transfected with T790A channels. $F$, Normalized current-voltage relationships of T790A-mediated resurgent currents. G, Summary of ratio resurgent current. C-G, All DRG neurons were held at $-80 \mathrm{mV}$ and pretreated with $500 \mathrm{~nm}$ TTX. Filled and open circles represent control and $\beta 4$ siRNA treatment, respectively. ${ }^{*} p<0.05$.

tude from $12.1 \pm 2.0 \%$ to $5.3 \pm 0.9 \%(p<0.05)$ of the peak transient current (Fig. $4 E, G$ ). Nav $\beta 4$ knockdown did not alter the current-voltage relationship of resurgent currents (Fig. $4 F$ ).

As DRG neurons also express several TTX-S sodium channel subtypes (e.g., Nav1.6, Nav1.7) that are able to produce TTX-S resurgent currents, and because those resurgent currents are altered by changing Nav $\beta 4$ subunit expression level to influence neuronal excitability (Barbosa et al., 2015), we added 500 nм TTX into the bath solution to block all TTX-S resurgent currents in the following current-clamp recordings, allowing us to focus on TTX-R-dependent action potential activity. Nav $\beta 4$ knockdown did not change resting membrane potential or current threshold (data not shown) and did not significantly reduce the proportion of T790A-transfected neurons with spontaneous firing. Under control conditions, $60.0 \%$ of L4-L5 DRG neurons with T790A mutant channels ( 12 of 20 cells) spontaneously generated action potentials, which is consistent with the observation in neurons isolated from all DRGs (Fig. 2B). After treatment with Nav $\beta 4$ siRNA, 9 of 20 transfected neurons (45.0\%) displayed spontaneous activity (Fig. $5 A, B$ ). This difference was not significant. However, as can be seen in Figure $5 C$, single evoked action potentials were substantially narrower after Nav $\beta 4$ siRNA treatment. The average durations measured under these two conditions were $253.8 \pm 93.4 \mathrm{~ms}$ and $38.1 \pm 8.2 \mathrm{~ms}(p<0.05)$, respectively (Fig. $5 D$, left). Under control conditions, $50 \%$ of transfected neurons ( 10 of 20 cells) generated single action potential with duration of $>50 \mathrm{~ms}$, but the percentage is only 10\% (2 of 20 cells) after treatment with Nav $\beta 4$ siRNA (Fig. $5 D$, right; $p<0.01$ ). The reduction indicated that an increase in Nav1.8-mediated resurgent currents can play a crucial role in broadening nociceptive neuron action potentials. With $2 \mathrm{~s}$ injected currents of $100 \mathrm{pA}$, T790A-transfected neurons treated with Nav $\beta 4$ siRNA displayed fewer action potentials than control T790A-transfected neurons
(Fig. $5 E-G$ ). The decrease in action potential number elicited was not significant at $200 \mathrm{pA}$. Overall, our data indicate that $\mathrm{Nav} \beta 4$ is not only important for generation of prolonged action potentials but also can impact firing frequency in nociceptive neurons transfected with T790A mutant channels.

\section{An SFN mutation in Nav1.8 that impairs inactivation increases resurgent current}

Next, we asked whether a human mutation, G1662S, identified in patients with painful SFN, endowed Nav1.8 with enhanced resurgent current generation. Han et al. (2014) reported that the SFN mutation impaired inactivation of human Nav1.8 expressed in mouse DRG neurons. Here we transfected rat DRG neurons with hNav1.8 WT and G1662S channel constructs. As shown in Figure $6 A, B$, the SFN mutation significantly slows fast inactivation of Nav1.8 current elicited by a $50 \mathrm{~ms}$ depolarizing potential of 10 $\mathrm{mV}$. At $10 \mathrm{mV}$, the fast-component time constant was $1.89 \pm$ $0.18 \mathrm{~ms}$ and $2.41 \pm 0.25 \mathrm{~ms}(p>0.05)$ for WT and G1662S hNav1.8 channels, respectively, whereas the slow-component value was $9.83 \pm 0.78 \mathrm{~ms}$ and $13.95 \pm 1.49 \mathrm{~ms}(p<0.05)$, respectively. The SFN mutation did not shift the voltage dependence of activation but induced a slight $(6.6 \mathrm{mV})$ positive shift in the voltage dependence of inactivation (Fig. 6D). Accordingly, the shift induced an increased overlap of the activation and inactivation curves, which is predicted to result in increased window currents (Fig. 6E). TTX-R resurgent currents were elicited using the same protocol as described for mNav1.8 and T790A mutant channels (Fig. 6F). Larger TTX-R resurgent currents were induced with G1662S mutant channels, compared with WT hNav1.8 (Fig. 6G). The average relative amplitude was $5.44 \pm$ $0.80 \%$ of peak transient currents, significantly larger than WT hNav1.8-mediated resurgent currents $(3.3 \pm 0.61 \%$; $p<0.05)$. The fraction of cells exhibiting detectable resurgent currents was 
A

control
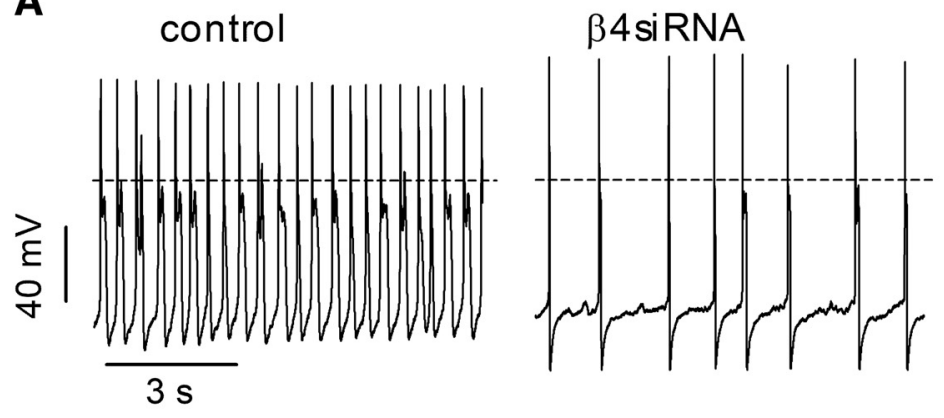

B

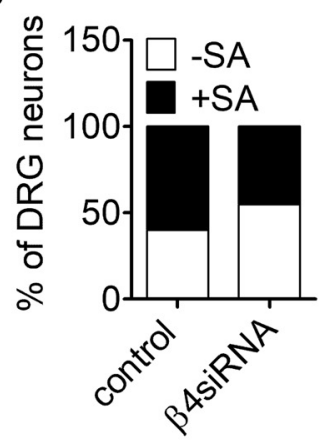

C

control

$\beta 4$ siRNA
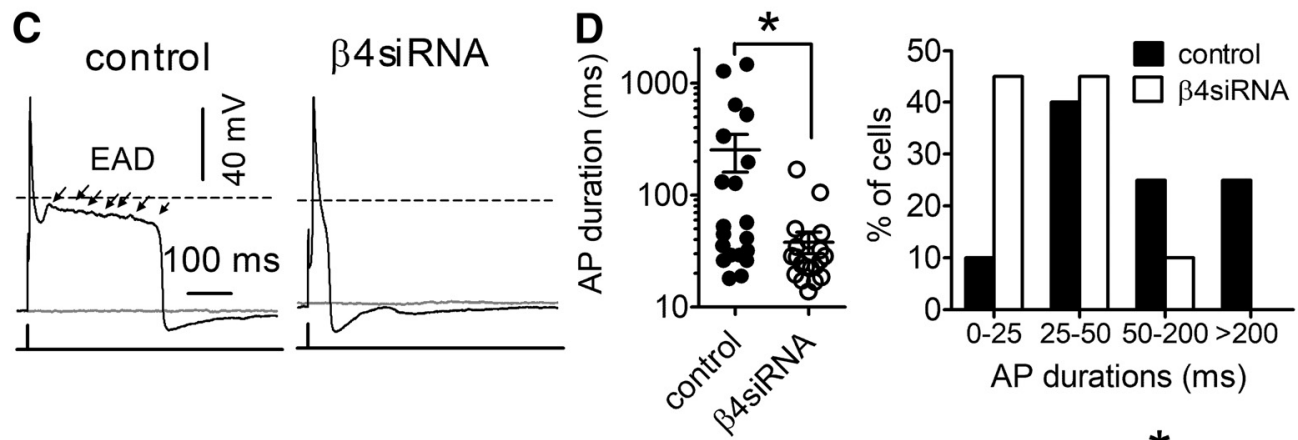

E

$0.1 \mathrm{nA}, 2 \mathrm{~s}$
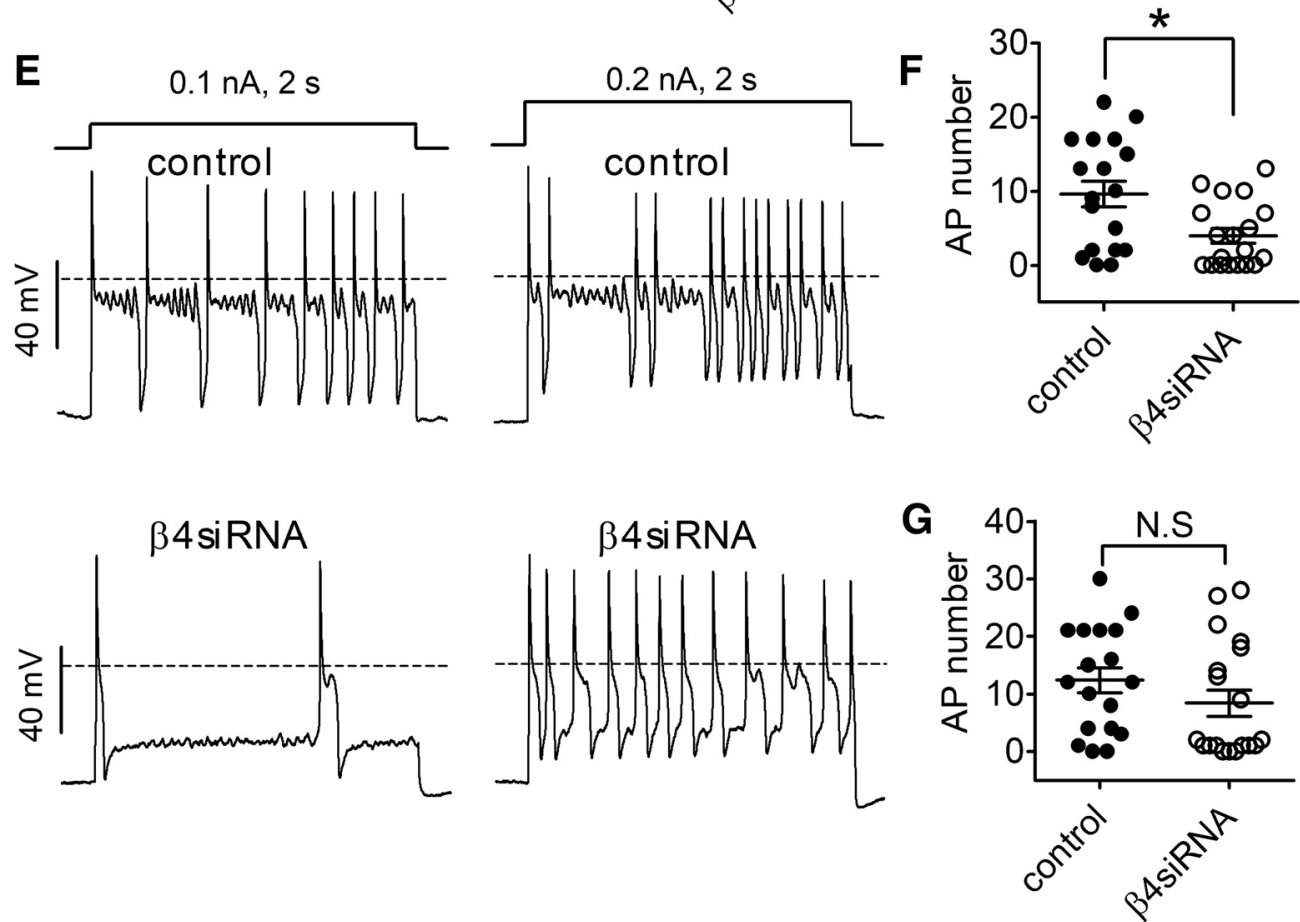

Figure 5. Knockdown of Nav $\beta 4$ subunit reduced excitability in L4-L5 DRG neurons transfected with T790A mutant channels. $A$, Typical spontaneous firing recorded from T790A-transfected DRG neurons with or without Nav $\beta 4$ siRNA treatment. $B$, Bar graph represents the percentage of DRG neurons with (white) or without (black) spontaneous firing. $+S A$, Spontaneous activity; $-S A$, no spontaneous activity. C, Typical single action potentials elicited by a $1 \mathrm{~ms}$ current injection. Arrows indicate EADs during the shoulder of the repolarization. $\boldsymbol{D}$, Summary of action potential duration (APD90). Left, Scatter plot of APD90. The average durations were $253.8 \pm 93.4 \mathrm{~ms}$ (control, $n=20)$ and $38.1 \pm 8.2 \mathrm{~ms}(\beta 4$ siRNA, $n=20)$. Right, Histogram showing the percentage of cells generating action potentials with the duration of $0-25,25-50,50-200$, and $>200 \mathrm{~ms}$. $\boldsymbol{E}$, Typical action potential trains elicited by a $2 \mathrm{~s}$ injection of 100 (left) or 200 (right) pA current. $\boldsymbol{F}, \boldsymbol{G}$, Summary of the number of action potentials elicited by a 2 s injection of 100 or $200 \mathrm{pA}$ current, respectively. $A, C, E$, Dotted line indicates zero voltage level. ${ }^{*} p<0.05$.

also greater with G1662S channels $(p<0.05$; Fig. $6 H$ ). The resurgent currents hNav1.8 and G1662S resurgent currents peaked at $-15 \mathrm{mV}$ and showed a slow onset and decay similar to those mediated by mav1.8 and T790A mutant channels (Fig. 6 F). The SFN mutation did not alter the voltage dependence of activation of TTX-R resurgent currents (Fig. 6I).
For comparison, we also introduced the human disease mutation G1662S into the mouse Nav1.8 ortholog; G1662 is equivalent to the Gly residue at position 1663 in mNav1.8. The G1663S mutation did not evidently affect the time constants of mNav1.8 inactivation $\left(\tau_{\text {fast }}:\right.$ mNav1.8, $2.4 \pm 0.2 \mathrm{~ms}, n=15$ vs G1663S, $2.6 \pm 0.2 \mathrm{~ms}, n=8, p>0.05 ; \tau_{\text {slow }}: \mathrm{mNav1} 1.8,11.2 \pm 1.1 \mathrm{~ms}$ vs 

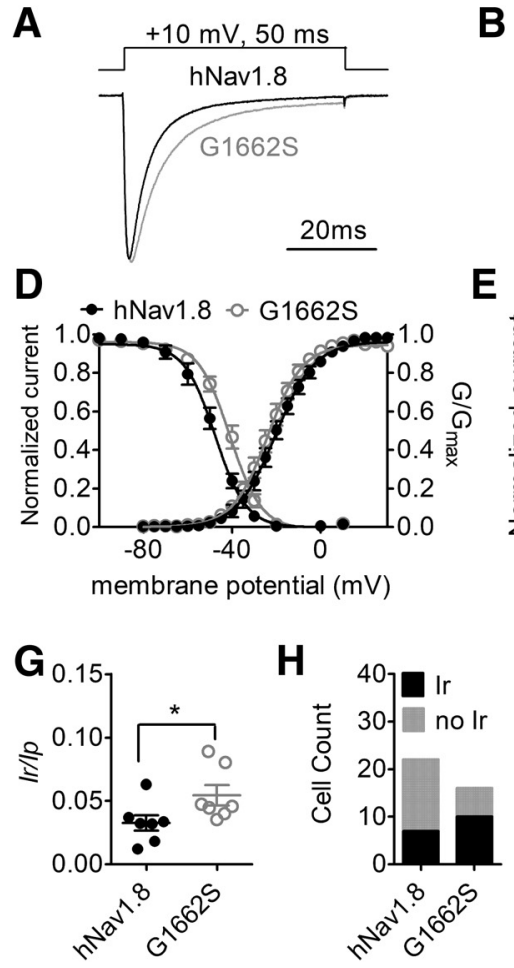

E
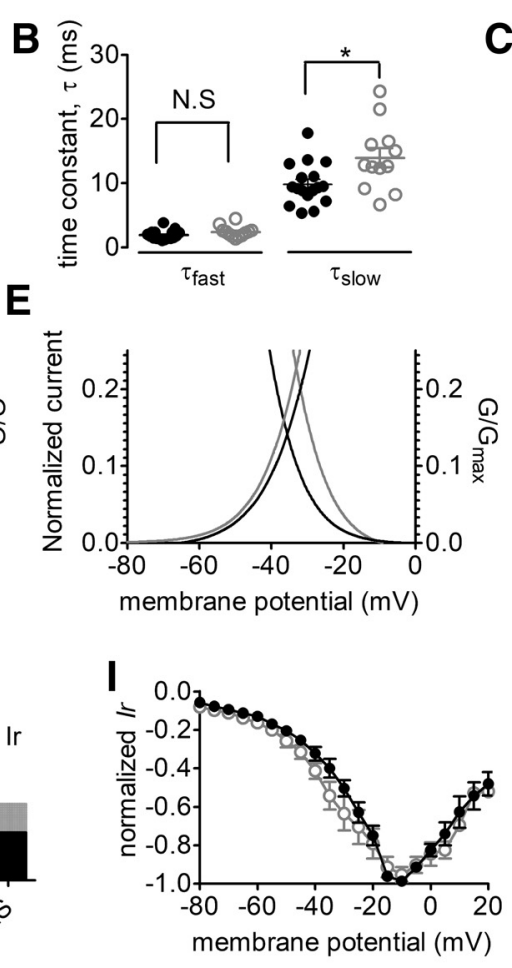

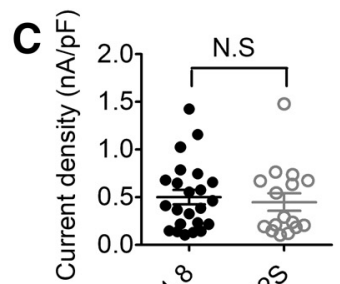

तथ ${ }^{0^{4}} 0^{0^{60^{2}}}$

$\mathbf{F}$

$+30 \mathrm{mV}$

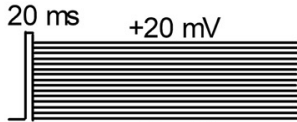

$80 \mathrm{mV}$

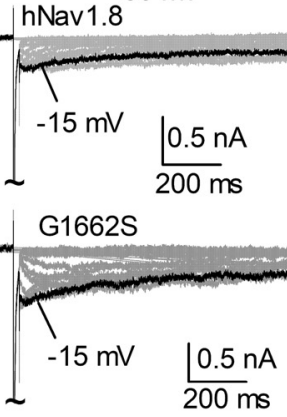

Figure 6. Effects of the $\mathrm{G} 1662 \mathrm{~S}$ mutation on hNav1.8 gating properties and hNav1.8-mediated resurgent currents at $21^{\circ} \mathrm{C}$. $\mathrm{A}$, The $\mathrm{G} 1662 \mathrm{~S}$ mutation slows the rate of fast inactivation. Currents were evoked by a $50 \mathrm{~ms}$ depolarizing potential of $10 \mathrm{mV}$. B, Summary of time constants of the fast and slow components of inactivation. Time constants were obtained by fitting with double exponential functions. G1662S significantly changed the slow time constant of fast inactivation at $10 \mathrm{mV}$. C, The G1662S mutation did not affect the current density. The averaged current density of WT hNav1.8 is $0.4997 \pm 0.07 \mathrm{nA} / \mathrm{pF}, n=23$; and the current density of hNav1.8 channel with G1662S mutation is $0.4477 \pm 0.09 \mathrm{nA} / \mathrm{pF}, n=16$. D, The G1662S mutation did not affect the voltage- conductance relationship but shifted steady-state inactivation of hNav1.8 to more positive potentials. Families of currents for $I-V$ curves and steady-state inactivation were induced using the same protocols as described in the Figure 1 C legend. Data points are mean \pm SE. $V_{1 / 2}$ for activation: hNav1.8, $-20.2 \pm 0.56 \mathrm{mV}, n=17 ; G 1662 S,-23.96 \pm 0.58 \mathrm{mV}, n=10$. $V_{1 / 2}$ for inactivation: $\mathrm{hNav1.8,}-47.64 \pm 1.06 \mathrm{mV}, n=16 ; \mathrm{G} 1662 \mathrm{~S},-41.06 \pm 0.60 \mathrm{mV}, n=12 . E$, Comparison of the overlap of steady-state activation and inactivation curves between $\mathrm{hNav1.8}$ (black curves) and G1662S (gray curves). $\boldsymbol{F}$, The G1662S mutation increased hNav1.8-mediated resurgent currents. Resurgent currents were induced with the protocol shown and described in the Figure

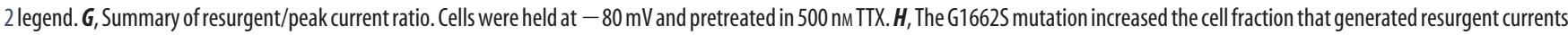
(hNav1.8, $n=23 ;$ G1662S, $n=16$ ). I, Comparison of normalized current-voltage relationships of hNav1.8- and G1662S-mediated resurgent currents. ${ }^{*} p<0.05$.

A

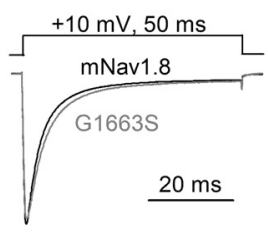

$\mathbf{B}_{8}$

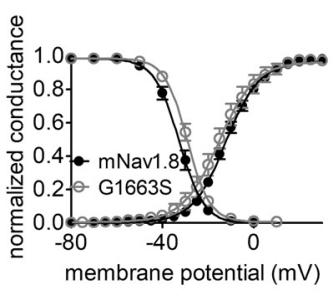

D $+30 \mathrm{mV}$,

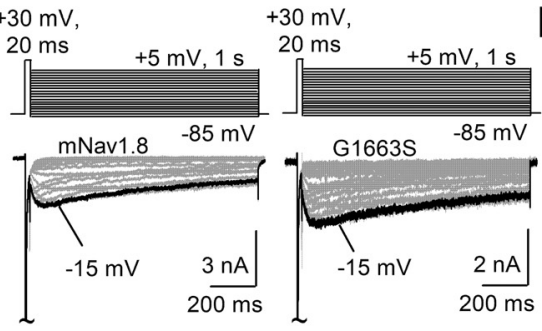

E

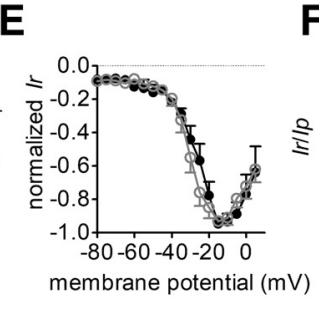

$\mathbf{F}$

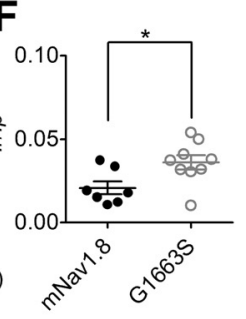

Figure 7. An SFN mutation of hNav1.8 alters mNav1.8 channel inactivation and resurgent currents at $21^{\circ} \mathrm{C}$. $\boldsymbol{A}$, The $\mathrm{G} 1663 \mathrm{~S}$ mutation did not significantly affect the rate of fast inactivation for mNav1.8. B. The G1663S mutation did not affect the voltage dependence of activation but induced a positive shift of steady-state inactivation of mNav1.8. $V_{1 / 2}$ for activation: mNav1.8, $-12.3 \pm 0.3 \mathrm{mV}, n=13 ; G 1663 \mathrm{~S},-15.3 \pm 0.7 \mathrm{mV}, n=8 . V_{1 / 2}$ for inactivation: $\mathrm{mNav1} .8,-32.7 \pm 0.5 \mathrm{mV}, n=13 ; \mathrm{G} 1663 \mathrm{~S}$, $-29.2 \pm 0.4 \mathrm{mV}, n=8$. C, G1663S mutation enhanced window current of mNav1.8. D, Representative mNav1.8 and G1663S resurgent current traces. The G1663S mutation increased mNav1.8-mediated resurgent current amplitudes. $\boldsymbol{E}$, Comparison of normalized current-voltage relationships of mNav1.8- and G1663S-mediated resurgent currents. $\boldsymbol{F}$, Comparison of resurgent current ratios from mNav1.8 and G1663S channels. ${ }^{*} p<0.05$.

G1663S, $12.5 \pm 2.7 \mathrm{~ms}, p>0.05$; Fig. $7 A)$. The G1663S mutation did not evidently alter the voltage dependence of mNav1.8 activation either but slightly impaired the voltage dependence of inactivation by 3.5 $\mathrm{mV}\left(V_{1 / 2}\right.$ for inactivation: mNav1.8, $-32.7 \pm 0.5 \mathrm{mV}, n=13$; G1663S, $-29.2 \pm 0.4 \mathrm{mV}, n=8 ; p<0.05$; Fig. $7 \mathrm{~B}, \mathrm{C})$. Our results show that the $\mathrm{G} 1663 \mathrm{~S}$ mutation also enhanced resurgent currents of mNav1.8 (Fig. 7D-F). The peak G1663S resurgent currents in absolute terms $(1.04 \pm 0.20 \mathrm{nA}, n=9)$ were twice as large as WT mNav1.8-mediated resurgent currents $(0.49 \pm 0.12 \mathrm{nA}, n=7 ; p<$ $0.05)$. The average relative amplitude was $3.61 \pm 0.42 \%$ of peak transient currents, also significantly larger than WT mNav1.8mediated resurgent currents $(2.08 \pm 0.40 \%$; $p<0.05$; Fig. $7 F$ ). These results are consistent with the observations in the human Nav1.8 construct (Fig. 6).

\section{Discussion}

Our data demonstrate, for the first time, that (1) human Nav1.8 channels can gen- 
erate resurgent sodium currents, (2) pathophysiological Nav1.8 mutations can significantly increase Nav1.8 resurgent currents, and (3) increased Nav1.8 openchannel block is likely to be an important contributor to nociceptive neuron hyperexcitability. In this study, we showed that TTX-R resurgent currents were increased by a gain-of-function Nav1.8 mutation in painful SFN and a mutation identified in the Possum transgenic mouse line that is associated with enhanced pain sensitivities. We demonstrate that increased Nav1.8-mediated resurgent currents not only enabled nociceptive neurons to generate highfrequency bursts of action potentials but also substantially broadened action potentials and generated multiple EADs. We also found that $\mathrm{Nav} \beta 4$ is a major determinant for resurgent current generation of Nav1.8. Nociceptor hyperexcitability is a crucial factor in altered pain phenotypes, and Nav1.8 resurgent currents are likely to contribute to neuropathic pain in SFN as well as inflammatory pain conditions.

Our data provide compelling evidence that Nav1.8 has the intrinsic ability to produce resurgent currents and accounts for the generation of endogenous TTX-R resurgent currents in DRG neurons (Tan et al., 2014). The endogenous rat TTX-R resurgent currents and those mediated by recombinant mNavl.8 and hNav1.8 are all resistant to TTX, display similar voltage dependence of activation, and, importantly, show a very slow onset and decay. These characteristics are quite different from resurgent currents mediated by other sodium channel subtypes (Jarecki et al., 2010; Barbosa et al., 2015). There is considerable evidence accumulating to suggest that open-channel block induced by $\mathrm{Nav} \beta 4$ is a major factor for TTX-S resurgent current generation. Our siRNA-mediated knockdown experiment indicates that Nav $\beta 4$ is also important for TTX-R resurgent current generation. The Nav $\beta 4$ cytoplasmic tail likely functions as an open-channel blocker, which directly competes with channel inactivation to dock transiently within (or nearby) the channel pore (Grieco et al., 2005). We predict that the large TTX-R resurgent currents detected in our expression system are also evoked in human DRG neurons. There is one residue that is different between the cytoplasmic tails of rat and human Nav $\beta 4$ (Yu et al., 2003), but this residue at position 188 (a Thr in rat and an Ile in human) is suggested to have little effect on Nav $\beta 4$-induced resurgent currents (Lewis and Raman, 2011). However, whereas we achieved close to $90 \%$ knockdown of Nav $\beta 4$ protein, Nav1.8 resurgent currents were only reduced by $56 \%$. This raises the possibility that other open-channel blockers, in addition to Nav $\beta 4$, interact with Nav1.8 channels and contribute to Nav1.8 resurgent currents.

The Possum T790A mutation impairs inactivation but also shifts activation by $-11.9 \mathrm{mV}$ in rat DRG neurons. This observation is different from those in a previous report, which indicated that the mutation does not modify the voltage dependence of Nav1.8 activation in the Possum transgenic mouse DRG neurons (Blasius et al., 2011). The activation shift identified in our study is unlikely due to different cell backgrounds. Such a shift was also observed when T790A channels were expressed in the ND7/23 cell line (Fig. 8). The hyperpolarized shift in activation is unlikely to contribute to enhanced resurgent currents but can also impact excitability. Interestingly, T790 is equivalent in position to T704 in human Nav1.4 and T698 in rat Nav1.4 sequences.
A mutation at this position in Nav1.4-(T704M) is associated with hyperkalemic periodic paralysis. Cummins et al. (1993) and Bendahhou et al. (1999) found that hNav1.4-T704M and rNav1.4T698M induce a hyperpolarizing shift in voltage dependence of Nav1.4 activation but do not impact inactivation. Our data demonstrate that this threonine residue, which is conserved across nine sodium channel isoforms, can influence both activation and inactivation gating of Nav1.8. This could represent an important difference in Nav1.8 gating compared with other sodium channel isoforms.

It is widely accepted that, whereas Nav1.7 helps set voltage threshold for action potential firing, Nav1.8 contributes mainly to the rising phase (Cummins et al., 2007). Many studies have shown that nociceptive sensory neurons generate action potentials with a prominent shoulder during the falling phase (Djouhri et al., 1998; López de Armentia et al., 2000). Blair and Bean (2002) demonstrated that TTX-R, not TTX-S, sodium current is active during this shoulder phase. Our present data demonstrate that an increase in TTX-R resurgent currents can substantially broaden the shoulder of the action potential. The broadening was greater at $34^{\circ} \mathrm{C}$. This likely reflects the resistance of overall Nav1.8 gating to temperature-induced changes (Zimmermann et al., 2007) coupled with the faster onset of Nav1.8 resurgent currents at warmer temperatures. Although the Possum mutation slows down fast inactivation and increases window currents, which presumably contributes to increased excitability, our $\mathrm{Nav} \beta 4$ knockdown experiments clearly indicated that the decrease in Nav1.8-mediated resurgent currents due to Nav $\beta 4$ knockdown results in shortened action potential durations, decreased EADs, and reduced firing frequency. Interestingly, Nav1.7 paroxysmal extreme pain disorder mutations slow fast inactivation and increase Nav1.7-mediated TTX-S resurgent currents, but paroxysmal extreme pain disorder mutations do not significantly broaden action potential duration (Dib-Hajj et al., 2008; Jarecki et al., 2010; Theile et al., 2011). We propose that, whereas Nav1.7mediated resurgent currents are mainly responsible for enhancing repetitive firing in nociceptive neurons, Nav1.8-mediated resurgent currents contribute to broadening of action potentials and to repetitive firing.

We also observed a substantial reduction in the rheobase (current threshold) for action potential firing with the T790A mutation. Bant and Raman (2010) demonstrated that Nav $\beta 4$-induced open-channel block of TTX-sensitive resurgent currents in cerebellar granule cells not only contributes to enhanced resurgent currents and increased repetitive firing, but also to increased persistent currents, a depolarized voltage dependence of inactiva- 
tion, and a lower rheobase. In our study, $\mathrm{Nav} \beta 4$ knockdown did not significantly impact rheobase in T790A-transfected cells. This could reflect the possible interaction of Nav1.8 an additional open-channel blocker. The change in rheobase with T790A is also likely, at least in part, to result from the mutation induced alterations in other biophysical properties, such as enhanced activation and impaired inactivation.

Our data indicate that hNav1.8 generates TTX-R resurgent currents almost twice as large as the mouse ortholog in rat DRG neurons. We did not observe a major difference in the inactivation properties of mouse and human Nav1.8 channels transfected in rat DRG neurons, so it is unclear precisely why hNav1.8 generated larger resurgent currents. The SFN mutation G1662S is the first human disease-causing mutation shown to enhance generation of TTX-R resurgent currents. This mutation increased Nav1.8-mediated resurgent currents by $65 \%$. In addition, whereas only $30 \%$ of neurons transfected with WT hNav1.8 generated resurgent currents, $63 \%$ of neurons transfected with G1662S did. It is likely that these changes in resurgent current amplitude and frequency are sufficient to increase nociceptive neuron excitability.

We propose that a fourfold increase in resurgent current amplitude, such as that observed with the Possum mutation, underlies the extreme neuronal hyperexcitability, aberrant pain sensitivity, and possibly even the freezing response to pinch in Possum mice (Blasius et al., 2011; Garrison et al., 2014). The SFN mutation had a smaller impact on resurgent current amplitude than the Possum mutation. A more moderate increase in resurgent currents is consistent with the more moderate increases in excitability reported for the SFN G1662S mutation (Han et al., 2014). In hNav1.8, the G1662S SFN mutation induced a significant slowing of inactivation. Although the change in fast inactivation time constant is similar for both the T790A and the G1662S mutations, the T790A mutation has a greater impact on late currents, and this difference likely contributes to the much larger resurgent currents with the Possum mutation. Lewis and Raman (2013) showed that open-channel blockers might have higher affinity in sodium channels with DIVS4 deployed than with DIVS4 in the resting or partially deployed configuration. We assume that T790A mutant channels exhibit a lower affinity for the open-channel blocker than WT Nav1.8 channels at voltages more negative than $-20 \mathrm{mV}$, and this coupled with augmented window currents results in increased current amplitudes at voltages between -25 and -45 $\mathrm{mV}$. Our data indicate that either increasing window currents or slowing fast inactivation is likely to enhance Nav1.8mediated resurgent current generation.

In conclusion, our findings provide novel mechanistic insight into how disease mutations alter generation of Nav1.8-mediated resurgent currents and how aberrant TTX-R resurgent currents regulate hyperexcitability of nociceptive sensory neurons. Our data demonstrate, for the first time, that painful Nav1.8 mutations induce aberrant TTX-R resurgent currents, and these are likely to serve as substantial contributors to the pathological mechanisms underlying nociceptive neuron hyperexcitability associated with neuropathic pain. We have previously shown that inflammatory mediators increase TTX-R resurgent currents (Tan et al., 2014). Thus, inhibition of TTX-R resurgent currents by pharmacological agents or reduction of Nav1.8 open-channel block by knocking down $\mathrm{Nav} \beta 4$ expression may present an effective strategy to treat neuropathic pain in SFN and other painful conditions.

\section{References}

Afshari FS, Ptak K, Khaliq ZM, Grieco TM, Slater NT, McCrimmon DR, Raman IM (2004) Resurgent Na currents in four classes of neurons of the cerebellum. J Neurophysiol 92:2831-2843. CrossRef Medline

Akopian AN, Sivilotti L, Wood JN (1996) A tetrodotoxin-resistant voltagegated sodium channel expressed by sensory neurons. Nature 379:257262. CrossRef Medline

Akopian AN, Souslova V, England S, Okuse K, Ogata N, Ure J, Smith A, Kerr BJ, McMahon SB, Boyce S, Hill R, Stanfa LC, Dickenson AH, Wood JN (1999) The tetrodotoxin-resistant sodium channel SNS has a specialized function in pain pathways. Nat Neurosci 2:541-548. CrossRef Medline

Bant JS, Raman IM (2010) Control of transient, resurgent, and persistent current by open-channel block by Na channel beta 4 in cultured cerebellar granule neurons. Proc Natl Acad Sci U S A 107:12357-12362. CrossRef Medline

Barbosa C, Tan ZY, Wang R, Xie W, Strong JA, Patel RR, Vasko MR, Zhang JM, Cummins TR (2015) Nav $\beta 4$ regulates fast resurgent sodium currents and excitability in sensory neurons. Mol Pain 11:60. CrossRef Medline

Barbosa C, Xiao Y, Johnson AJ, Xie W, Strong JA, Zhang JM, Cummins TR (2017) FHF2 isoforms differentially regulate Nav1.6-mediated resurgent sodium currents in dorsal root ganglion neurons. Pflugers Arch 469:195212. CrossRef Medline

Bendahhou S, Cummins TR, Tawil R, Waxman SG, Ptácek LJ (1999) Activation and inactivation of the voltage-gated sodium channel: role of segment $\mathrm{S} 5$ revealed by a novel hyperkalaemic periodic paralysis mutation. J Neurosci 19:4762-4771. CrossRef Medline

Blair NT, Bean BP (2002) Roles of tetrodotoxin (TTX)-sensitive $\mathrm{Na}^{+}$current, TTX-resistant $\mathrm{Na}^{+}$current, and $\mathrm{Ca}^{2+}$ current in the action potentials of nociceptive sensory neurons. J Neurosci 22:10277-10290. CrossRef Medline

Blair NT, Bean BP (2003) Role of tetrodotoxin-resistant $\mathrm{Na}^{+}$current slow inactivation in adaptation of action potential firing in small-diameter dorsal root ganglion neurons. J Neurosci 23:10338-10350. CrossRef Medline

Blasius AL, Dubin AE, Petrus MJ, Lim BK, Narezkina A, Criado JR, Wills DN, Xia Y, Moresco EM, Ehlers C, Knowlton KU, Patapoutian A, Beutler B (2011) Hypermorphic mutation of the voltage-gated sodium channel encoding gene Scn10a causes a dramatic stimulus-dependent neurobehavioral phenotype. Proc Natl Acad Sci US A 108:19413-19418. CrossRef Medline

Cannon SC, Bean BP (2010) Sodium channels gone wild: resurgent current from neuronal and muscle channelopathies. J Clin Invest 120:80-83. CrossRef Medline

Cummins TR, Zhou J, Sigworth FJ, Ukomadu C, Stephan M, Ptácek LJ, Agnew WS (1993) Functional consequences of a $\mathrm{Na}^{+}$channel mutation causing hyperkalemic periodic paralysis. Neuron 10:667-678. CrossRef Medline

Cummins TR, Howe JR, Waxman SG (1998) Slow closed-state inactivation: a novel mechanism underlying ramp currents in cells expressing the hNE/ PN1 sodium channel. J Neurosci 18:9607-9619. CrossRef Medline

Cummins TR, Black JA, Dib-Hajj SD, Waxman SG (2000) Glial-derived neurotrophic factor upregulates expression of functional SNS and NaN sodium channels and their currents in axotomized dorsal root ganglion neurons. J Neurosci 20:8754-8761. CrossRef Medline

Cummins TR, Dib-Hajj SD, Waxman SG (2004) Electrophysiological properties of mutant Nav1.7 sodium channels in a painful inherited neuropathy. J Neurosci 24:8232-8236. CrossRef Medline

Cummins TR, Dib-Hajj SD, Herzog RI, Waxman SG (2005) Nav1.6 channels generate resurgent sodium currents in spinal sensory neurons. FEBS Lett 579:2166-2170. CrossRef Medline

Cummins TR, Sheets PL, Waxman SG (2007) The roles of sodium channels in nociception: implications for mechanisms of pain. Pain 131:243-257. CrossRef Medline

Dib-Hajj SD, Cummins TR, Black JA, Waxman SG (2007) From genes to pain: Na v 1.7 and human pain disorders. Trends Neurosci 30:555-563. CrossRef Medline

Dib-Hajj SD, Estacion M, Jarecki BW, Tyrrell L, Fischer TZ, Lawden M, Cummins TR, Waxman SG (2008) Paroxysmal extreme pain disorder M1627K mutation in human Nav1.7 renders DRG neurons hyperexcitable. Mol Pain 4:37. CrossRef Medline

Dib-Hajj SD, Choi JS, Macala LJ, Tyrrell L, Black JA, Cummins TR, Waxman 
SG (2009) Transfection of rat or mouse neurons by biolistics or electroporation. Nat Protoc 4:1118-1126. CrossRef Medline

Djouhri L, Bleazard L, Lawson SN (1998) Association of somatic action potential shape with sensory receptive properties in guinea-pig dorsal root ganglion neurones. J Physiol 513:857-872. CrossRef Medline

Enomoto A, Han JM, Hsiao CF, Wu N, Chandler SH (2006) Participation of sodium currents in burst generation and control of membrane excitability in mesencephalic trigeminal neurons. J Neurosci 26:3412-3422. CrossRef Medline

Faber CG, Hoeijmakers JG, Ahn HS, Cheng X, Han C, Choi JS, Estacion M, Lauria G, Vanhoutte EK, Gerrits MM, Dib-Hajj S, Drenth JP, Waxman SG, Merkies IS (2012a) Gain of function Na $\nu 1.7$ mutations in idiopathic small fiber neuropathy. Ann Neurol 71:26-39. CrossRef Medline

Faber CG, Lauria G, Merkies IS, Cheng X, Han C, Ahn HS, Persson AK, Hoeijmakers JG, Gerrits MM, Pierro T, Lombardi R, Kapetis D, Dib-Hajj SD, Waxman SG (2012b) Gain-of-function Nav1.8 mutations in painful neuropathy. Proc Natl Acad Sci U S A 109:19444-19449. CrossRef Medline

Fang X, McMullan S, Lawson SN, Djouhri L (2005) Electrophysiological differences between nociceptive and non-nociceptive dorsal root ganglion neurones in the rat in vivo. J Physiol 565:927-943. CrossRef Medline

Fertleman CR, Baker MD, Parker KA, Moffatt S, Elmslie FV, Abrahamsen B, Ostman J, Klugbauer N, Wood JN, Gardiner RM, Rees M (2006) SCN9A mutations in paroxysmal extreme pain disorder: allelic variants underlie distinct channel defects and phenotypes. Neuron 52:767-774. CrossRef Medline

Garrison SR, Weyer AD, Barabas ME, Beutler BA, Stucky CL (2014) A gainof-function voltage-gated sodium channel 1.8 mutation drives intense hyperexcitability of A- and C-fiber neurons. Pain 155:896-905. CrossRef Medline

Grieco TM, Raman IM (2004) Production of resurgent current in NaV1.6null Purkinje neurons by slowing sodium channel inactivation with betapompilidotoxin. J Neurosci 24:35-42. CrossRef Medline

Grieco TM, Malhotra JD, Chen C, Isom LL, Raman IM (2005) Openchannel block by the cytoplasmic tail of sodium channel beta 4 as a mechanism for resurgent sodium current. Neuron 45:233-244. CrossRef Medline

Han C, Vasylyev D, Macala LJ, Gerrits MM, Hoeijmakers JG, Bekelaar KJ, Dib-Hajj SD, Faber CG, Merkies IS, Waxman SG (2014) The G1662S NaV1.8 mutation in small fibre neuropathy: impaired inactivation underlying DRG neuron hyperexcitability. J Neurol Neurosurg Psychiatry 85: 499-505. CrossRef Medline

Hasegawa Y, Mao W, Saha S, Gunner G, Kolpakova J, Martin GE, Futai K (2017) Luciferase shRNA presents off-target effects on voltage-gated ion channels in mouse hippocampal pyramidal neurons. eNeuro 4:ENEURO.0186-17.2017. CrossRef Medline

Herzog RI, Cummins TR, Ghassemi F, Dib-Hajj SD, Waxman SG (2003) Distinct repriming and closed state inactivation kinetics of Navl.6 and Nav1.7 sodium channels in mouse spinal sensory neurons. J Physiol 551: 741-750. CrossRef Medline

Hoeijmakers JG, Merkies IS, Gerrits MM, Waxman SG, Faber CG (2012) Genetic aspects of sodium channelopathy in small fiber neuropathy. Clin Genet 82:351-358. CrossRef Medline

Huang J, Yang Y, Zhao P, Gerrits MM, Hoeijmakers JG, Bekelaar K, Merkies IS, Faber CG, Dib-Hajj SD, Waxman SG (2013) Small-fiber neuropathy Nav1.8 mutation shifts activation to hyperpolarized potentials and increases excitability of dorsal root ganglion neurons. J Neurosci 33:1408714097. CrossRef Medline

Jackson AL, Linsley PS (2010) Recognizing and avoiding siRNA off-target effects for target identification and therapeutic application. Nat Rev Drug Discov 9:57-67. CrossRef Medline

Jarecki BW, Piekarz AD, Jackson JO 2nd, Cummins TR (2010) Human voltage-gated sodium channel mutations that cause inherited neuronal and muscle channelopathies increase resurgent sodium currents. J Clin Invest 120:369-378. CrossRef Medline

Kim JH, Kushmerick C, von Gersdorff H (2010) Presynaptic resurgent $\mathrm{Na}^{+}$ currents sculpt the action potential waveform and increase firing reliability at a CNS nerve terminal. J Neurosci 30:15479-15490. CrossRef Medline

Lewis AH, Raman IM (2011) Cross-species conservation of open-channel block by Na channel $\beta 4$ peptides reveals structural features required for resurgent Na current. J Neurosci 31:11527-11536. CrossRef Medline

Lewis AH, Raman IM (2013) Interactions among DIV voltage-sensor movement, fast inactivation, and resurgent $\mathrm{Na}$ current induced by the NaVß4 open-channel blocking peptide. J Gen Physiol 142:191-206. CrossRef Medline

López de Armentia M, Cabanes C, Belmonte C (2000) Electrophysiological properties of identified trigeminal ganglion neurons innervating the cornea of the mouse. Neuroscience 101:1109-1115. CrossRef Medline

Mikami M, Yang J (2005) Short hairpin RNA-mediated selective knockdown of NaV1.8 tetrodotoxin-resistant voltage-gated sodium channel in dorsal root ganglion neurons. Anesthesiology 103:828-836. CrossRef Medline

Nuyens D, Stengl M, Dugarmaa S, Rossenbacker T, Compernolle V, Rudy Y, Smits JF, Flameng W, Clancy CE, Moons L, Vos MA, Dewerchin M, Benndorf K, Collen D, Carmeliet E, Carmeliet P (2001) Abrupt rate accelerations or premature beats cause life-threatening arrhythmias in mice with long-QT3 syndrome. Nat Med 7:1021-1027. CrossRef Medline

Patel RR, Barbosa C, Xiao Y, Cummins TR (2015) Human Nav1.6 channels generate larger resurgent currents than human Nav1.1 channels, but the Nav $\beta 4$ peptide does not protect either isoform from use-dependent reduction. PLoS One 10:e0133485. CrossRef Medline

Raman IM, Bean BP (1997) Resurgent sodium current and action potential formation in dissociated cerebellar Purkinje neurons. J Neurosci 17: 4517-4526. CrossRef Medline

Raman IM, Sprunger LK, Meisler MH, Bean BP (1997) Altered subthreshold sodium currents and disrupted firing patterns in Purkinje neurons of Scn8a mutant mice. Neuron 19:881-891. CrossRef Medline

Ritter DM, Zemel BM, Lepore AC, Covarrubias M (2015) Kv3.4 channel function and dysfunction in nociceptors. Channels (Austin) 9:209-217. CrossRef Medline

Song W, Xiao Y, Chen H, Ashpole NM, Piekarz AD, Ma P, Hudmon A, Cummins TR, Shou W (2012) The human Nav1.5 F1486 deletion associated with long QT syndrome leads to impaired sodium channel inactivation and reduced lidocaine sensitivity. J Physiol 590:5123-5139. CrossRef Medline

Tan ZY, Piekarz AD, Priest BT, Knopp KL, Krajewski JL, McDermott JS, Nisenbaum ES, Cummins TR (2014) Tetrodotoxin-resistant sodium channels in sensory neurons generate slow resurgent currents that are enhanced by inflammatory mediators. J Neurosci 34:7190-7197. CrossRef Medline

Theile JW, Cummins TR (2011) Recent developments regarding voltagegated sodium channel blockers for the treatment of inherited and acquired neuropathic pain syndromes. Front Pharmacol 2:54. CrossRef Medline

Theile JW, Jarecki BW, Piekarz AD, Cummins TR (2011) Nav1.7 mutations associated with paroxysmal extreme pain disorder, but not erythromelalgia, enhance Navbeta4 peptide-mediated resurgent sodium currents. J Physiol 589:597-608. CrossRef Medline

Themistocleous AC, Ramirez JD, Serra J, Bennett DL (2014) The clinical approach to small fibre neuropathy and painful channelopathy. Pract Neurol 14:368-379. CrossRef Medline

Xie W, Strong JA, Ye L, Mao JX, Zhang JM (2013) Knockdown of sodium channel NaV1.6 blocks mechanical pain and abnormal bursting activity of afferent neurons in inflamed sensory ganglia. Pain 154:1170-1180. CrossRef Medline

Xie W, Tan ZY, Barbosa C, Strong JA, Cummins TR, Zhang JM (2016) Upregulation of the sodium channel $\mathrm{NaV} \beta 4$ subunit and its contributions to mechanical hypersensitivity and neuronal hyperexcitability in a rat model of radicular pain induced by local DRG inflammation. Pain 157:879-891. CrossRef Medline

Yu FH, Westenbroek RE, Silos-Santiago I, McCormick KA, Lawson D, Ge P, Ferriera H, Lilly J, DiStefano PS, Catterall WA, Scheuer T, Curtis R (2003) Sodium channel $\beta 4$, a new disulfide-linked auxiliary subunit with similarity to $\beta 2$. J Neurosci 23:7577-7585. CrossRef Medline

Zimmermann K, Leffler A, Babes A, Cendan CM, Carr RW, Kobayashi J, Nau C, Wood JN, Reeh PW (2007) Sensory neuron sodium channel Nav1.8 is essential for pain at low temperatures. Nature 447:855-858. CrossRef Medline 